\title{
INVARIANT MARKOV SEMIGROUPS ON QUANTUM HOMOGENEOUS SPACES
}

\author{
BISWARUP DAS, UWE FRANZ, AND XUMIN WANG
}

\begin{abstract}
Invariance properties of linear functionals and linear maps on algebras of functions on quantum homogeneous spaces are studied, in particular for the special case of expected coideal *-subalgebras. Several one-to-one correspondences between such invariant functionals are established. Adding a positivity condition, this yields one-to-one correspondences of invariant quantum Markov semigroups acting on expected coideal *-subalgebras and certain convolution semigroups of states on the underlying compact quantum group. This gives an approach to classifying invariant quantum Markov semigroups on these quantum homogeneous spaces. The generators of these semigroups are viewed as Laplace operators on these spaces.

The classical sphere $S^{N-1}$, the free sphere $S_{+}^{N-1}$, and the half-liberated sphere $S_{*}^{N-1}$ are considered as examples and the generators of Markov semigroups on these spheres a classified. We compute spectral dimensions for the three families of spheres based on the asymptotic behaviour of the eigenvalues of their Laplace operator.
\end{abstract}

\section{INTRODUCTION}

Symmetry plays an essential role in many places in mathematics and in the natural sciences. Many systems are naturally invariant under the action of some group, like time or space translations, rotations, or reflections. It is therefore of great interest to characterize and classify all invariant equations for a given group action. See for example the recent books by Ming Liao [L18] and Vladimir Dobrev [D16], that study invariant Markov processes and invariant differential operators, respectively. Liao's book is motivated by probability theory, whereas Dobrev's book deals with applications to physics.

Quantum groups [W80, W87] provide a generalisation of groups and can be considered as a mathematical model for quantum symmetries. Dobrev [D17] has also studied invariant differential operators for quantum groups. The quantum groups considered in [D17] are q-deformations of semi-simple Lie groups.

But there exist also interesting quantum groups that are not deformations, but rather "liberations" of classical groups, see, e.g., [VDW96, W98, BS09. These "liberated" quantum groups furthermore have actions on interesting "liberated"

2000 Mathematics Subject Classification. 46L53 17B37 17B81 46L65 60B15 60 G51 81R50 .

Key words and phrases. Compact quantum group, quantum homogeneous space, quantum Markov semigroup, free sphere, Laplace operator. 
noncommutative spaces, see, e.g., [BG10]. This provides an interesting class of examples for noncommutative geometry.

Banica and Goswami investigated how to define a Dirac operator on two of these noncommutative spaces: the free sphere $S_{+}^{N-1}$ and the half-liberated sphere $S_{\times}^{N-1}$, cf. [BG10, Theorem 6.4]. The action of the free or the half-liberated orthogonal group yields a natural choice for the eigenspaces, but it does not suggest how to choose the eigenvalues.

In this paper we introduce an approach for classifying invariant Markov semigroups on noncommutative spaces equipped with an action of a compact quantum group. The generators of these semigroups can be considered as natural candidates for Laplace operators. Dirac operators could be obtained via Cipriani and Sauvageot's construction CS03 of a derivation from a Dirichlet form, see also CFK14. Our method generalizes the case of an action of a classical compact group on a homogeneous space presented in [L04, Chapter 3], [L15], [L18, Chapter 1]. Since here we are dealing only with compact quantum groups and actions on compact quantum spaces, everything can be done on the ${ }^{*}$-algebraic level. As concrete examples we study the classical sphere $S^{N-1}$, the half-liberated sphere $S_{*}^{N-1}$, and the free sphere $S_{+}^{N-1}$.

Our approach adds a positivity condition to the invariance condition in [BG10], and leads to the formula

$$
\lambda_{k}=-b P_{k}^{\prime}(1)+\int_{-1}^{1} \frac{P_{k}(x)-P_{k}(1)}{x-1} \mathrm{~d} \nu(x), \quad k=0,1, \ldots,
$$

for the eigenvalues of the Laplace operator on the three spheres $S^{N-1}, S_{*}^{N-1}$, $S_{+}^{N-1}$, see Theorem 7.5 . Here $b$ is a positive real number, $\nu$ is a finite positive measure on the interval $[-1,1]$, and $\left(P_{k}\right)_{k=0}^{\infty}$ is a family of orthogonal polynomials that depends on which sphere we are considering.

We define spectral dimensions the three spheres by comparing the asymptotic behaviour of the eigenvalues of their Laplace operators to the Weyl formula. More precisely, the spectral dimension is defined as the abscissa of convergence of a certain zeta function defined in terms of the eigenvalues of the Laplace operator. We find, as expected, $d_{L}=N-1$ for the classical sphere $S^{N-1}$. For the half-liberated sphere $S_{*}^{N-1}$, we get $d_{L}=2(N-1)$. For the free sphere $S_{+}^{N-1}$, we obtain

$$
d_{L}=\left\{\begin{array}{cc}
2 & \text { if } N=2 \\
+\infty & \text { if } N \geq 3
\end{array}\right.
$$

It should be noted that, for $N=2$, the half-liberated sphere $S_{*}^{1}$ and the free sphere $S_{+}^{1}$ are isomorphic. A more detailed study of the zeta function could probably be used to introduce further interesting "invariants" for these noncommutative manifolds.

We now provide a brief description for the content of each section.

In Section 1, we recall some definitions and facts about quantum group actions, quantum quotient spaces, idempotent states, and quantum Markov semigroups 
Section 2 gives an overview of the actions and the notions of invariance that we will consider. Proposition 2.3 shows that convolution by a central functional defines an invariant operator.

In Section 3, we state and prove one-to-one correspondences between various invariant linear functionals and maps on a quantum homogeneous space and on the associated compact quantum group. In the following section we use these results to characterize invariant Markov semigroups on expected right coidalgebras, cf. Section 4 .

Bi-invariance leads to examples of so-called quantum hypergroups, cf. ChV99, and in Section 5 we show that invariant Markov semigroups on expected right coidalgebras are in one-to-one correspondence with convolution semigroups of states on a quantum hypergroup that is naturally associated to the coidalgebra.

Section 6 provides a short summary of our main one-to-one correspondences.

The general theory developped in Sections 2, 3, 4, and 5, allows us to classify the generators of invariant Markov semigroups on quantum homogeneous spaces that are associated to an idempotent state $\Phi$ on the underlying compact quantum group $\mathbb{G}$, in particular if we have a good understanding of the quantum hypergroup $\Phi \backslash \mathbb{G} / \Phi$. This is slightly more general than in the classical case, where all homogeneous spaces are of quotient type, but follows similar ideas.

In Section 7. we apply our approach to classify invariant Markov semigroups on the classical sphere $S^{N-1}$, on the half-liberated sphere $S_{*}^{N-1}$, and on the free

sphere $S_{+}^{N-1}$. In Theorem 7.5 we give the general form of the eigenvalues of the generators of these semigroups. In the rest of Section 7 we study in more detail the orthogonal polynomials that occur in this formula. We also show in Proposition 7.1 that the the half-liberated sphere $S_{*}^{N-1}$ and the free sphere $S_{+}^{N-1}$ are not of quotient type. In Subsection 7.4 we define a zeta function in terms of the eigenvalues of generators we classified before, determine its abscissa of convergence, and compute from this the spectral dimensions of the spheres.

We think it would be interesting to extend this study to other expected quantum homogeneous spaces, e.g., those of Banica and Speicher "easy" compact quantum groups [BS09], where many combinatorial techniques are available for explicit calculations. And it would of course be very useful to develop methods that also apply for not necessarily expected quantum homogeneous spaces.

Conventions: We use $\otimes$ both for the tensor product of vector spaces and $*_{-}$ algebras, and for the minimal tensor product of $\mathrm{C}^{*}$-algebras, the meaning will be clear from the context.

\section{Preliminaries}

1.1. Compact quantum groups. For an introduction to the theory of compact quantum groups, see W98, MVD98, T08. 
1.2. Actions of compact quantum groups. We adopt the convention that for a compact quantum group $\mathbb{G}, C^{u}(\mathbb{G})$ denotes the unital $\mathrm{C}^{*}$-algebra of the universal version of $\mathbb{G}$, whereas $C(\mathbb{G})$ denotes that of the reduced version. We refer the reader to [DC16] for a recent survey on actions of compact quantum groups.

Definition 1.1. A right action $\mathbb{X} \stackrel{\alpha}{\curvearrowleft} \mathbb{G}$ of a compact quantum group $\mathbb{G}$ on a compact quantum space $X$ (also called a right coaction of $C(\mathbb{G})$ on the unital $\mathrm{C}^{*}$-algebra $\left.C(\mathbb{X})\right)$ is a unital *-homomorphism

$$
\alpha: C(\mathbb{X}) \rightarrow C(\mathbb{X}) \otimes C(\mathbb{G})
$$

such that

- the coaction property holds:

$$
\left(\alpha \otimes \operatorname{id}_{C(\mathbb{G})}\right) \circ \alpha=\left(\operatorname{id}_{C(\mathbb{X})} \otimes \Delta\right) \circ \alpha
$$

and

- the density condition (also called Podleś condition)

$$
\overline{\alpha(C(\mathbb{X}))\left(\mathbf{1}_{C(\mathbb{X})} \otimes C(\mathbb{G})\right)}=C(\mathbb{X}) \otimes C(\mathbb{G})
$$

holds.

Associated with every right action of a compact quantum group $\mathbb{G}$ on a compact quantum space $\mathbb{X}$ is the Podleś subalgebra or the algebraic core of $C(\mathbb{X})$, which we denote by $\mathscr{O}_{\mathbb{G}}(\mathbb{X})$. We refer to [DC16, pp. $\left.25-27\right]$ for a detailed description of the properties of $\mathscr{O}_{\mathbb{G}}(\mathbb{X})$. We collect a few facts for $\mathscr{O}_{\mathbb{G}}(\mathbb{X})$ :

- Considering $\mathbb{G} \curvearrowleft \mathbb{G}$ by the coproduct, the corresponding Podleś subalgebra (or Peter-Weyl algebra) $\mathscr{O}(\mathbb{G})$ is precisely the unique, dense Hopf *-algebra $\mathscr{O}(\mathbb{G})$ of $\mathbb{G}$, which is also commonly denoted by $\operatorname{Pol}(\mathbb{G})$. It is spanned by the coefficients of the finite-dimensional corepresentations of $C(\mathbb{G})$.

- $\mathscr{O}_{\mathbb{G}}(\mathbb{X}) \subset C(\mathbb{X})$ is a dense, unital * subalgebra of $C(\mathbb{X})$ DC16, Theorem 3.16].

- The right coaction $\alpha: C(\mathbb{X}) \longrightarrow C(\mathbb{X}) \otimes C(\mathbb{G})$ restricts to a right Hopf *-coaction $\mathscr{O}(\mathbb{G})$ on the unital $*$ algebra $\mathscr{O}_{\mathbb{G}}(\mathbb{X})$ :

$$
\left.\alpha\right|_{\mathscr{O}_{\mathbb{G}}(\mathbb{X})}: \mathscr{O}_{\mathbb{G}}(\mathbb{X}) \longrightarrow \mathscr{O}_{\mathbb{G}}(\mathbb{X}) \otimes \mathscr{O}(\mathbb{G})
$$

An action is called embeddable, if $\mathscr{O}_{\mathbb{G}}(\mathbb{X})$ is isomorphic to a ${ }^{*}$-subalgebra of $\mathscr{O}(\mathbb{G})$, such that the action corresponds to the restriction of the coproduct, i.e., if there exists an injective unital *-homomorphism $\vartheta: \mathscr{O}_{\mathbb{G}}(\mathbb{X}) \rightarrow \mathscr{O}(\mathbb{G})$ such that $\left.(\vartheta \otimes \mathrm{id}) \circ \alpha\right|_{\mathscr{O}_{\mathbb{G}}(\mathbb{X})}=\Delta \circ \vartheta$. Such actions can be given as unital ${ }_{\text {-subalgebras }}$ which are also coideals.

Definition 1.2. A left (right, resp.) coidalgebra of $\mathscr{O}(\mathbb{G})$ is unital *-subalgebra $\mathcal{C}$ of $\mathscr{O}(\mathbb{G})$ such that

$$
\Delta(\mathcal{C}) \subseteq \mathcal{C} \otimes \mathscr{O}(\mathbb{G}), \quad(\Delta(\mathcal{C}) \subseteq \mathscr{O}(\mathbb{G}) \otimes \mathcal{C} \text { resp. })
$$


1.3. Quantum quotient space. Let $\mathbb{K}$ be a compact quantum subgroup of $\mathbb{G}$, which we will take to mean:

- $\mathbb{K}$ is a compact quantum group.

- There exists a surjective, unital *-homomorphism $\theta: C^{u}(\mathbb{G}) \longrightarrow C^{u}(\mathbb{K})$ such that

$$
(\theta \otimes \theta) \circ \Delta_{u}=\Delta_{u, \mathbb{K}} \circ \theta
$$

where $\Delta_{u}$ is the coproduct of $C^{u}(\mathbb{G})$ and $\Delta_{u, \mathbb{K}}$ is the coproduct of $C^{u}(\mathbb{K})$. Then the $C^{*}$-algebra of the left quantum quotient of $\mathbb{G}$ by $\mathbb{K}$, denoted $C^{u}(\mathbb{K} \backslash \mathbb{G})$ is defined as

$$
C^{u}(\mathbb{K} \backslash \mathbb{G}):=\left\{x \in C^{u}(\mathbb{G}):(\theta \otimes \mathrm{id})\left(\Delta_{u}(x)\right)=1_{C^{u}(\mathbb{K})} \otimes x\right\} .
$$

$C^{u}(\mathbb{K} \backslash \mathbb{G})$ consists of the elements of $C^{u}(\mathbb{G})$ that are invariant under the left action $(\theta \otimes \mathrm{id}) \circ \Delta_{u}: C^{u}(\mathbb{G}) \rightarrow C^{u}(\mathbb{K}) \otimes C^{u}(\mathbb{G})$ of $\mathbb{K}$ on $\mathbb{G}$ induced by $\theta$.

We collect a few facts about the subalgebra $C^{u}(\mathbb{K} \backslash \mathbb{G})$ below, see also DC16, P95:

- $\Delta_{u}\left(C^{u}(\mathbb{K} \backslash \mathbb{G})\right) \subset C^{u}(\mathbb{K} \backslash \mathbb{G}) \otimes C^{u}(\mathbb{G})$. Letting $\Lambda: C^{u}(\mathbb{G}) \longrightarrow C(\mathbb{G})$ be the reducing morphism,

$$
\alpha:=\left.(\mathrm{id} \otimes \Lambda) \circ \Delta\right|_{C^{u}(\mathbb{K} \backslash \mathbb{G})}: C^{u}(\mathbb{K} \backslash \mathbb{G}) \longrightarrow C^{u}(\mathbb{K} \backslash \mathbb{G}) \otimes C(\mathbb{G})
$$

is a right action of $\mathbb{G}$ on $C^{u}(\mathbb{K} \backslash \mathbb{G})$.

- $\mathscr{O}_{\mathbb{G}}(\mathbb{K} \backslash \mathbb{G}) \subset \mathscr{O}(\mathbb{G})$ and it can be easily seen that $\left.\alpha\right|_{\mathscr{O}_{\mathbb{G}}(\mathbb{K} \backslash \mathbb{G})}=\left.\Delta_{u}\right|_{\mathscr{O}_{\mathbb{G}}(\mathbb{K} \backslash \mathbb{G})}$. Thus letting $W \in M\left(C(\mathbb{G}) \otimes C_{0}(\widehat{\mathbb{G}})\right)$ be the left multiplicative unitary, it follows that

$$
\alpha(x)=W^{*}\left(1_{\mathbb{G}} \otimes x\right) W \quad\left(x \in \mathscr{O}_{\mathbb{G}}(\mathbb{K} \backslash \mathbb{G})\right) .
$$

Denoting the norm closure of $\mathscr{O}_{\mathbb{G}}(\mathbb{K} \backslash \mathbb{G})$ in $C(\mathbb{G})$ by $C(\mathbb{K} \backslash \mathbb{G})$, it follows from the above equation that

$$
\left.\Delta\right|_{C(\mathbb{K} / \mathbb{G})}: C(\mathbb{K} / \mathbb{G}) \longrightarrow C(\mathbb{K} \backslash \mathbb{G}) \otimes C(\mathbb{G})
$$

is a right action of $\mathbb{G}$ on $C(\mathbb{K} \backslash \mathbb{G})$, which restricted to $\mathscr{O}_{\mathbb{G}}(\mathbb{K} \backslash \mathbb{G})$ is the right

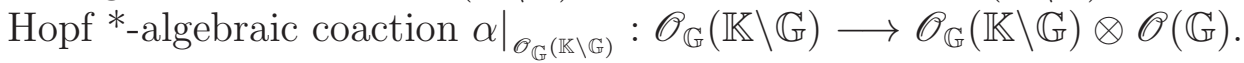

1.4. Idempotent states. In this paper we will be interested in actions coming from idempotent states, as in the following theorem.

Theorem 1.3. (FS09a and FLS16]) Let $\mathbb{G}$ be a compact quantum group. There is a one-to-one correspondence between the following objects:

(1) idempotent states $\Phi$ on $\mathscr{O}(\mathbb{G})$;

(2) idempotent states $\tilde{\Phi}$ on $C^{u}(\mathbb{G})$;

(3) expected right (equivalently, left) coidalgebras $\mathcal{A}$ in $\mathscr{O}(\mathbb{G})$ (denote by $\mathcal{E}$ : $\mathscr{O}(\mathbb{G}) \rightarrow \mathcal{A}$ the conditional expectation);

(4) expected right (equivalently, left) coidalgebras $A$ in $C(\mathbb{G})$ (denote by $E$ : $C(\mathbb{G}) \rightarrow$ A the conditional expectation). 
The one-to-one correspondence is given by the following relations: $\tilde{\Phi}$ is a continuous extension of $\Phi$, and $\mathcal{A}=(\mathrm{id} \otimes \Phi) \circ \Delta(\mathscr{O}(\mathbb{G}))$. The $\mathrm{C}^{*}$-algebra $\mathrm{A}$ is the norm closure of $\mathcal{A}$ in $C(\mathbb{G})$. On $\mathscr{O}(\mathbb{G})$ we can recover the idempotent state as $\Phi=\varepsilon \circ \mathcal{E}$. Moreover, each of the maps $E$ and $\mathcal{E}$ preserves the Haar state.

We wil denote by $\mathcal{I}(\mathbb{G})$ the set of idempotent states on $C^{u}(\mathbb{G})$. In view of the one-to-one correspondence in Theorem [1.3, we will denote by $\mathcal{A}_{\Phi}$ and $\mathrm{A}_{\Phi}$ the right coidalgebras associated to $\Phi \in \mathcal{I}(\mathbb{G})$, and from now on we will denote by $\mathbb{E}_{r}^{\Phi}$ the conditional expecations both onto $\mathcal{A}_{\Phi}$ and onto $\mathrm{A}_{\Phi}$ in $\mathscr{O}(\mathbb{G})$ or $C(\mathbb{G})$, respectively. On $\mathscr{O}(\mathbb{G})$ this conditional expectation can be defined by the formula $\mathbb{B}_{r}^{\Phi}=(\mathrm{id} \otimes \Phi) \circ \Delta$. The correspondence in Theorem 1.3 preserves the natural order, i.e., we have

$$
\Phi_{1} \star \Phi_{2}=\Phi_{1} \quad \Leftrightarrow \quad \mathcal{A}_{\Phi_{1}} \subseteq \mathcal{A}_{\Phi_{2}} \quad \Leftrightarrow \quad \mathrm{A}_{\Phi_{1}} \subseteq \mathrm{A}_{\Phi_{2}},
$$

since $\mathbb{E}_{r}^{\Phi_{1}} \circ \mathbb{E}_{r}^{\Phi_{2}}=\left(\mathrm{id} \otimes\left(\Phi_{2} \star \Phi_{2}\right)\right) \circ \Delta=\left.\mathbb{E}\right|_{r} ^{\Phi_{1} \star \Phi_{2}}$.

Theorem 1.3 has recently been generalized to locally compact quantum groups, see [SS16, KK16].

Recalling the definition of quantum quotient spaces as given in the previous subsection, it is worthwhile to note the following:

- Let $h_{\mathbb{K}}$ be the Haar state on $C^{u}(\mathbb{K})$. Then $\Phi_{\mathbb{K}}=h_{\mathbb{K}} \circ \theta \in \mathcal{I}(\mathbb{G})$, and it follows that $C^{u}(\mathbb{K} \backslash \mathbb{G})$ is the right coidalgebra of $C^{u}(\mathbb{G})$ associated with $\Phi_{\mathbb{K}}=h_{\mathbb{K}} \circ \theta$.

- Letting $E_{\mathbb{K} \backslash \mathbb{G}}:=\left(h_{\mathbb{K}} \circ \theta \otimes \mathrm{id}\right) \circ \Delta_{u}$ and $E_{\mathbb{G} / \mathbb{K}}:=\left(\mathrm{id} \otimes h_{\mathbb{K}} \circ \theta\right) \circ \Delta_{u}$ (both are conditional expectations), the unital ${ }^{*}$-subalgebra $E_{\mathbb{K} / \mathbb{G}}(\mathscr{O}(\mathbb{G})) \cap E_{\mathbb{G} / \mathbb{K}}(\mathscr{O}(\mathbb{G}))$ is a double coset hyper bi-algebra, as considered in [FS00].

For our set-up, we will be mainly concerned with expected right coidalgebras of $\mathbb{G}$ (we remark that analogous results hold for left coidalgebras). As pointed out above quantum quotient spaces are special cases of these. We may note that expected right coidalgebras of $\mathbb{G}$ are examples of quantum homogeneous spaces, i.e. quantum spaces on which the corresponding right action of $\mathbb{G}$ is ergodic [P95].

\subsection{Convolution semigroups of states and quantum Markov semigroups} on compact quantum groups. We recall a few handy definitions and facts from CFK14.

Definition 1.4. A convolution semigroup on a compact quantum group $\mathbb{G}$ is a family $\left(\lambda_{t}\right)_{t \geq 0}: \mathscr{O}(\mathbb{G}) \rightarrow \mathbb{C}$ such that

(1) $\lim _{t \searrow_{0}} \lambda_{t}(a)=\lambda_{0}(a)$ for all $a \in \mathscr{O}(\mathbb{G})$ (weak continuity);

(2) $\lambda_{s} \star \lambda_{t}=\lambda_{s+t}$ for all $s, t \geq 0$ (semigroup property).

We call $\left(\lambda_{t}\right)_{t \geq 0}$ a convolution semigroup of states, if the functionals $\lambda_{t}$ are furthermore normalized, i.e., $\lambda_{t}(1)=1$, and positive, i.e., $\lambda_{t}\left(a^{*} a\right) \geq 0$ for all $a \in \mathscr{O}(\mathbb{G})$ and all $t \geq 0$.

The semigroup property implies that $\lambda_{0}$ is idempotent, but note that unlike [CFK14 we do not require $\lambda_{0}=\varepsilon$. The convolution semigroups on $\mathbb{G}$ that we 
will obtain from Markov semigroups on $\mathbb{G}$-spaces will in general not start with the counit.

Definition 1.5. A linear operator $T: A \rightarrow A$ on a unital $C^{*}$-algebra $A$ is called a quantum Markov operator, if it is completely positive and preserves the unit of A.

A quantum Markov semigroup on $\mathrm{A}$ is a family $\left(T_{t}\right)_{t \geq 0}$ of Markov operators satisfying

(1) $\lim _{t \searrow 0} T_{t}(a)=T_{0}(a)$ in norm for all $a \in \mathrm{A}$ (pointwise norm-continuity);

(2) $T_{s} \circ T_{t}=T_{s+t}$ for all $s, t \geq 0$ (semigroup property).

A linear operator $T: \mathcal{A} \rightarrow \mathcal{A}$ (or a family of linear operators $\left(T_{t}: \mathcal{A} \rightarrow \mathcal{A}\right)_{t \geq 0}$, resp.) on a unital ${ }^{*}$-algebra $\mathcal{A}$ is called a quantum Markov operator (semigroup, resp.), if it is the restriction of a quantum Markov operator (semigroup, resp.) on a $\mathrm{C}^{*}$-algebra $\mathrm{A}$ containing $\mathcal{A}$ that preserves $\mathcal{A}$.

In [CFK14, Theorem 3.2] it was shown that for a convolution semigroup of states $\left(\lambda_{t}\right)_{t>0}$ with $\lambda_{0}=\varepsilon$ on a compact quantum group there always exists a unique quantum Markov semigoup $\left(T_{t}\right)_{t \geq 0}$ (with $T_{0}=\mathrm{id}$ ) on $C(\mathbb{G})$ that acts on elements $a \in \mathscr{O}(\mathbb{G})$ of the Hopf *-algebra as

$$
T_{t}(a)=\left(\mathrm{id} \otimes \lambda_{t}\right) \circ \Delta(a) .
$$

Quantum Markov semigroups coming in this way from convolution semigroups of states are characterized by the invariance property $\left.\Delta \circ T_{t}=\left(\mathrm{id} \otimes T_{t}\right) \circ \Delta\right)$, cf. CFK14, Theorem 3.4].

\section{ACTIONS AND INVARIANCES}

Let us start in the algebraic setting. A functional $\phi \in \mathscr{O}(\mathbb{G})^{\prime}$ can act in three ways on another functional $f \in \mathscr{O}(\mathbb{G})^{\prime}$ :

$$
\begin{aligned}
L_{\phi} f & =\phi \star f, \\
R_{\phi} f & =f \star \phi, \\
\operatorname{Ad}_{\phi} f & : \mathscr{O}(\mathbb{G}) \ni a \mapsto \phi\left(a_{(1)} S\left(a_{(3)}\right)\right) f\left(a_{(2)}\right) \in \mathbb{C},
\end{aligned}
$$

and by duality it can also act in three ways on an element $a \in \mathscr{O}(\mathbb{G})$ :

$$
\begin{aligned}
L_{\phi}^{*} a & =\phi\left(a_{(1)}\right) a_{(2)}, \\
R_{\phi}^{*} a & =\phi\left(a_{(2)}\right) a_{(1)}, \\
\operatorname{Ad}_{\phi}^{*} a & =\phi\left(a_{(1)} S\left(a_{(3)}\right)\right) a_{(2)} .
\end{aligned}
$$

It is straightforward to check that we have

$$
L_{\phi_{1}} \circ L_{\phi_{2}}=L_{\phi_{1} \star \phi_{2}}, \quad R_{\phi_{1}} \circ R_{\phi_{2}}=R_{\phi_{2} \star \phi_{1}}
$$

and

$$
L_{\phi_{1}}^{*} \circ L_{\phi_{2}}^{*}=L_{\phi_{2} \star \phi_{1}}^{*}, \quad R_{\phi_{1}}^{*} \circ R_{\phi_{2}}^{*}=R_{\phi_{1} \star \phi_{2}}^{*}
$$


for $\phi_{1}, \phi_{2} \in \mathscr{O}(\mathbb{G})^{\prime}$. Furthermore,

$$
\operatorname{Ad}_{\phi_{1}}^{*}\left(\operatorname{Ad}_{\phi_{2}}^{*}(a)\right)=\phi_{2}\left(a_{(1)}\right) \phi_{1}\left(a_{(2)}\right) \phi_{1}\left(S\left(a_{(4)}\right)\right) \phi_{2}\left(S\left(a_{(5)}\right)\right) a_{(3)}=\operatorname{Ad}_{\phi_{1} \star \phi_{2}}^{*}(a)
$$

for $\phi_{1}, \phi_{2} \in \mathscr{O}(\mathbb{G})^{\prime}, a \in \mathscr{O}(\mathbb{G})$, and

$$
\operatorname{Ad}_{\phi_{1}} \circ \operatorname{Ad}_{\phi_{2}}=\operatorname{Ad}_{\phi_{1} \star \phi_{2}} .
$$

If $\phi \in\left(\mathscr{O}(\mathbb{G})^{\prime}\right.$ is positive, then it extends to a unique positive functional on $C^{u}(\mathbb{G})$, cf. [BMT01, Theorem 3.3]. In this case its actions $L_{\phi}^{*}$ and $R_{\phi}^{*}$ on $\mathscr{O}(\mathbb{G})$ extend continuously to unique completely positive maps on $C(\mathbb{G})$ and $C^{u}(\mathbb{G})$, see, e.g., [Br12, Lemma 3.4]. $L_{\phi}^{*}$ and $R_{\phi}^{*}$ are furthermore unital iff $\phi$ is a state, i.e., if $\phi(\mathbf{1})=1$.

Definition 2.1. For a subset $M \subseteq \mathscr{O}(\mathbb{G})^{\prime}$ we define the spaces of left $M$-invariant, $\underline{\text { right } M \text {-invariant, and adjoint } M \text {-invariant functionals and polynomial functions }}$ as

$$
\begin{aligned}
\left(\mathscr{O}(\mathbb{G})^{\prime}\right)^{L(M)} & =\left\{f \in \mathscr{O}(\mathbb{G})^{\prime} ; \forall \phi \in M, L_{\phi}(f)=\phi(\mathbf{1}) f\right\}, \\
\left(\mathscr{O}(\mathbb{G})^{\prime}\right)^{R(M)} & =\left\{f \in \mathscr{O}(\mathbb{G})^{\prime} ; \forall \phi \in M, R_{\phi}(f)=\phi(\mathbf{1}) f\right\}, \\
\left(\mathscr{O}(\mathbb{G})^{\prime}\right)^{\operatorname{Ad}(M)} & =\left\{f \in \mathscr{O}(\mathbb{G})^{\prime} ; \forall \phi \in M, \operatorname{Ad}_{\phi}(f)=\phi(\mathbf{1}) f\right\}, \\
\mathscr{O}(\mathbb{G})^{L^{*}(M)} & =\left\{a \in \mathscr{O}(\mathbb{G}) ; \forall \phi \in M, L_{\phi}^{*}(a)=\phi(\mathbf{1}) a\right\}, \\
\mathscr{O}(\mathbb{G})^{R^{*}(M)} & =\left\{a \in \mathscr{O}(\mathbb{G}) ; \forall \phi \in M, R_{\phi}^{*}(a)=\phi(\mathbf{1}) a\right\}, \\
\mathscr{O}(\mathbb{G})^{\operatorname{Ad}^{*}(M)} & =\left\{a \in \mathscr{O}(\mathbb{G}) ; \forall \phi \in M, \operatorname{Ad}_{\phi}^{*}(a)=\phi(\mathbf{1}) a\right\} .
\end{aligned}
$$

The conjugate $M$-invariant functionals and polynomial functions are

$$
\begin{aligned}
\left(\mathscr{O}(\mathbb{G})^{\prime}\right)^{\operatorname{Conj}(M)} & =\left\{f \in \mathscr{O}(\mathbb{G})^{\prime} ; \forall \phi \in M, L_{\phi}(f)=R_{\phi}(f)\right\}, \\
\mathscr{O}(\mathbb{G})^{\text {Conj }^{*}(M)} & =\left\{a \in \mathscr{O}(\mathbb{G}) ; \forall \phi \in M, L_{\phi}^{*}(a)=R_{\phi}^{*}(a)\right\} .
\end{aligned}
$$

For $M=\mathscr{O}(\mathbb{G})^{\prime}$ they are also called central functionals and polynomial functions.

We also define a notion of invariance for functionals and linear operators on quantum homogeneous spaces.

Definition 2.2. Let $\alpha: \mathscr{O}_{\mathbb{G}}(\mathbb{X}) \rightarrow \mathscr{O}_{\mathbb{G}}(\mathbb{X}) \otimes \mathscr{O}(\mathbb{G})$ be a Hopf *-algebraic right action of a compact quantum group $\mathbb{G}$. We say that a linear map $T: \mathscr{O}_{\mathbb{G}}(\mathbb{X}) \rightarrow$ $\mathscr{O}_{\mathbb{G}}(\mathbb{X})$ is $\underline{\mathbb{G} \text {-invariant, }}$, if

$$
\alpha \circ T=(T \otimes \mathrm{id}) \circ \alpha .
$$

Let give us a first general construction of $\mathbb{G}$-invariant operators and Markov semigroups on a homogenous space.

Proposition 2.3. Let $\alpha: \mathscr{O}_{\mathbb{G}}(\mathbb{X}) \rightarrow \mathscr{O}_{\mathbb{G}}(X) \otimes \mathscr{O}(\mathbb{G})$ be a right action of a compact quantum group $\mathbb{G}$.

If $\phi: \mathscr{O}(\mathbb{G}) \rightarrow \mathbb{C}$ is a central functional, then $T_{\phi}=(\mathrm{id} \otimes \phi) \circ \alpha: \mathscr{O}_{\mathbb{G}}(\mathbb{X}) \rightarrow \mathscr{O}_{\mathbb{G}}(\mathbb{X})$ is $\mathbb{G}$-invariant. 
If $\left(\varphi_{t}\right)_{t \geq 0}$ is a central convolution semigroup of states on $\mathscr{O}(\mathbb{G})$ with $\varphi_{0}=\varepsilon$, then $T_{t}=\left(\mathrm{id} \otimes \varphi_{t}\right) \circ \alpha$ defines a $\mathbb{G}$-invariant quantum Markov semigroup with $T_{0}=\mathrm{id}$ on $\mathscr{O}_{\mathbb{G}}(\mathbb{X})$.

Proof. A functional $\phi: \mathscr{O}(\mathbb{G}) \rightarrow \mathbb{C}$ is central iff

$$
(\mathrm{id} \otimes \phi) \circ \Delta=(\phi \otimes \mathrm{id}) \circ \Delta .
$$

Therefore if $\phi$ is central, then we have

$$
\begin{aligned}
\alpha \circ T_{\phi} & =(\mathrm{id} \otimes \mathrm{id} \otimes \phi) \circ(\alpha \otimes \mathrm{id}) \circ \alpha \\
& =(\mathrm{id} \otimes \mathrm{id} \otimes \phi) \circ(\mathrm{id} \otimes \Delta) \circ \alpha \\
& =(\mathrm{id} \otimes \phi \otimes \mathrm{id}) \circ(\mathrm{id} \otimes \Delta) \circ \alpha \\
& =\left(T_{\phi} \otimes \mathrm{id}\right) \circ \alpha,
\end{aligned}
$$

as claimed. On the algebraic core we have $T_{0}=(\mathrm{id} \otimes \varepsilon) \circ \alpha=\mathrm{id}$.

The second statement follows, since the positivity of the $\varphi_{t}$ implies that the $T_{t}$ are completely positive, $T_{t}(\mathbf{1})=\mathbf{1} \varphi_{t}(\mathbf{1})=\mathbf{1}$ for all $t \geq 0$, and

$$
T_{t}(x)=x_{(0)} \varphi_{t}\left(x_{(1)}\right) \stackrel{t \searrow 0}{\longrightarrow} x_{(0)} \varepsilon\left(x_{(1)}\right)=x \quad \text { in norm }
$$

for $x \in \mathscr{O}_{q} g(\mathbb{X})$, by continuity of the convolution semigroup $\left(\varphi_{t}\right)_{t \geq 0}$, see also the proof of [CFK14, Theorem 3.2]. Here we used Sweedler notation $\alpha(x)=x_{(0)} \otimes x_{(1)}$ for the action.

\section{INVARIANT FUNCTIONALS, OPERATORS AND THEIR CONVOLUTIONS}

In this section we fix an idempotent state $\Phi \in \mathcal{I}(\mathbb{G})$ and suppose $C^{r}(\mathbb{G} / \Phi)$, $C^{r}(\Phi \backslash \mathbb{G}), \mathscr{O}_{\mathbb{G}}(\mathbb{G} / \Phi)$ and $\mathscr{O}_{\mathbb{G}}(\Phi \backslash \mathbb{G})$ denote the respective right and left coidalgebras. $\mathbb{E}_{r}^{\Phi}$ and $\mathbb{E}_{\ell}^{\Phi}$ denote respectively the conditional expectations from $\mathscr{O}(\mathbb{G})$ onto $\mathscr{O}_{\mathbb{G}}(\mathbb{G} / \Phi)$ and $\mathscr{O}_{\mathbb{G}}(\Phi \backslash \mathbb{G})$. And we use the same notations $\mathbb{E}_{r}^{\Phi}$ and $\mathbb{E}_{\ell}^{\Phi}$ for the conditional expectations from $C(\mathbb{G})$ onto $C^{r}(\mathbb{G} / \Phi)$ and $C^{r}(\Phi \backslash \mathbb{G})$. We may note that the restriction of the coproduct $\Delta$ to $\mathscr{O}_{\mathbb{G}}(\Phi \backslash \mathbb{G})$ and $\mathscr{O}_{\mathbb{G}}(\mathbb{G} / \Phi)$ are respectively left and right Hopf*-algebraic coactions of $\mathscr{O}(\mathbb{G})$ on $\mathscr{O}_{\mathbb{G}}(\Phi \backslash \mathbb{G})$ and $\mathscr{O}_{\mathbb{G}}(\mathbb{G} / \Phi)$. We start with two lemmas which we will be using in the sequel.

Lemma 3.1. On $\mathscr{O}(\mathbb{G})$ the following holds:

(a) $\left(\mathbb{E}_{\ell}^{\Phi} \otimes \mathrm{id}\right) \circ \Delta=\Delta \circ \mathbb{E}_{\ell}^{\Phi} ;\left(\mathrm{id} \otimes \mathbb{E}_{r}^{\Phi}\right) \circ \Delta=\Delta \circ \mathbb{E}_{r}^{\Phi}$.

(b) $\left(\mathbb{E}_{r}^{\Phi} \otimes \mathrm{id}\right) \circ \Delta=\left(\mathrm{id} \otimes \mathbb{E}_{\ell}^{\Phi}\right) \circ \Delta$.

Proof. The identity in (a) is actually the invariance condition for the conditional expectations, as observed in [FLS16].

We prove (b): 


$$
\begin{aligned}
\left(\mathbb{E}_{r}^{\Phi} \otimes \mathrm{id}\right) \circ \Delta & =((\mathrm{id} \otimes \Phi) \circ \Delta \otimes \mathrm{id}) \circ \Delta \\
& =(\mathrm{id} \otimes \Phi \otimes \mathrm{id}) \circ(\Delta \otimes \mathrm{id}) \circ \Delta \\
& =(\mathrm{id} \otimes \Phi \otimes \mathrm{id}) \circ(\mathrm{id} \otimes \Delta) \circ \Delta \\
& =(\mathrm{id} \otimes(\Phi \otimes \mathrm{id}) \circ \Delta) \circ \Delta=\left(\mathrm{id} \otimes \mathbb{E}_{\ell}^{\Phi}\right) \circ \Delta .
\end{aligned}
$$

The following is a minor variation of the result already observed in FS09b, Section 3].

Lemma 3.2. Let $\Phi_{1}, \Phi_{2}$ be idempotent states on $\mathscr{O}(\mathbb{G})$. If $\Phi_{1} * \Phi_{2}=\Phi_{2}$, then $\Phi_{2} * \Phi_{1}=\Phi_{2}$.

Proof. Let $S$ be the antipode of $\mathbb{G}$. For any idempotent state $\phi$ on $\mathscr{O}(\mathbb{G})$ we have $\phi \circ S=\phi$, see [FS09b, Section 3, pp. 10], or [SS16, Proposition 4], where this is shown even for locally compact quantum groups.

This along with the identity $(S \otimes S) \circ \Delta=\Delta^{\text {op }} \circ S$ immediately implies the desired result.

3.1. Invariant functionals on expected right coidalgebras. We will write $\alpha:=\left.\Delta\right|_{\mathscr{O}_{\mathbb{G}}(\Phi \backslash \mathbb{G})}$.

Definition 3.3. For $\Phi \in \mathcal{I}(\mathbb{G})$, we call a functional $f$ on $\mathscr{O}_{\mathbb{G}}(\Phi \backslash \mathbb{G}) \underline{\Phi \text {-invariant }}$ if $(f \otimes \Phi) \circ \alpha=f$.

We call a functional $f$ on $\mathscr{O}(\mathbb{G}) \Phi$-bi-invariant if $f \in\left(\mathscr{O}(\mathbb{G})^{\prime}\right)^{L(\{\Phi\})} \cap\left(\mathscr{O}(\mathbb{G})^{\prime}\right)^{R(\{\Phi\})}$.

Theorem 3.4. The following holds

(a) Let $f$ be a $\Phi$-invariant functional on $\mathscr{O}_{\mathbb{G}}(\Phi \backslash \mathbb{G})$. Then the functional defined by $\mu:=f \circ \mathbb{E}_{\ell}^{\Phi}$ is the unique $\Phi$-bi-invariant functional on $\mathscr{O}(\mathbb{G})$, whose restriction to $\mathscr{O}_{\mathbb{G}}(\Phi \backslash \mathbb{G})$ is $f$.

(b) Let $\mu$ be a $\Phi$-bi-invariant functional on $\mathscr{O}(\mathbb{G})$. Then $f:=\left.\mu\right|_{\mathscr{O}_{\mathbb{G}}(\Phi \backslash \mathbb{G})}$ is the unique $\Phi$-invariant functional on $\mathscr{O}_{\mathbb{G}}(\Phi \backslash \mathbb{G})$, such that $f \circ \mathbb{E}_{\ell}^{\Phi}=\mu$.

Proof. We prove (a):

Let $x \in \mathscr{O}(\mathbb{G})$. We prove the $\Phi$-bi-invariance of $\mu$ as follows:

Left $\Phi$-invariance:

$$
\begin{aligned}
(\Phi \otimes \mu)(\Delta(x)) & =\left(\Phi \otimes f \circ \mathbb{E}_{\ell}^{\Phi}\right)(\Delta(x)) \\
& =(\Phi \otimes f)\left(\left(\mathbb{E}_{r}^{\Phi} \otimes \mathrm{id}\right)(\Delta(x))\right) \quad(\text { by Lemma 3.1 }-(b)) \\
& =(\Phi \otimes \Phi \otimes f)(\Delta \otimes \mathrm{id})(\Delta(x)) \\
& =(\Phi * \Phi \otimes f)(\Delta(x)) \\
& =(\Phi \otimes f)(\Delta(x))=f\left(\mathbb{E}_{\ell}^{\Phi}(x)\right) \quad(\text { since } \Phi \in \mathcal{I}(\mathbb{G})) \\
& =\mu(x) .
\end{aligned}
$$


Right $\Phi$-invariance:

$$
\begin{aligned}
(\mu \otimes \Phi)(\Delta(x)) & =\left(f \circ \mathbb{E}_{\ell}^{\Phi} \otimes \Phi\right)(\Delta(x)) \\
& =(f \otimes \Phi)\left(\mathbb{E}_{\ell}^{\Phi} \otimes \mathrm{id}\right)(\Delta(x)) \\
& \left.=(f \otimes \Phi) \Delta\left(\mathbb{E}_{\ell}^{\Phi}(x)\right) \quad \text { (by Lemma 3.1- }(a)\right) \\
& \left.=f\left(\mathbb{E}_{\ell}^{\Phi}(x)\right) \quad \text { (using } \Phi \text {-invariance of } f\right) \\
& =\mu(x) .
\end{aligned}
$$

Now, let $\nu$ be any $\Phi$-bi-invariant functional on $\mathscr{O}(\mathbb{G})$ such that $\left.\nu\right|_{\mathscr{O}_{\mathbb{G}}(\Phi \backslash \mathbb{G})}=f$. Then using the right $\Phi$-invariance of $\nu$ we have

$$
\begin{aligned}
\nu(x) & =(\Phi \otimes \nu)(\Delta(x)) \\
& =\nu\left(\mathbb{E}_{\ell}^{\Phi}(x)\right) \\
& =f\left(\mathbb{E}_{\ell}^{\Phi}(x)\right)=\mu(x),
\end{aligned}
$$

which proves the uniqueness.

(b) follows by observing that the $\Phi$-invariance of $f$ as a functional on $\mathscr{O}_{\mathbb{G}}(\Phi \backslash \mathbb{G})$ is a consequence of the left $\Phi$-invariance of $\mu$ as a functional on $\mathscr{O}(\mathbb{G})$, and uniqueness can be seen easily.

Thus we have a one-to-one correspondence between the set of $\Phi$-invariant functional on $\mathscr{O}_{\mathbb{G}}(\Phi \backslash \mathbb{G})$ and that of $\Phi$-bi-invariant functionals on $\mathscr{O}(\mathbb{G})$. In particular, we have

Corollary 3.5. There exists a one-to-one correspondence between the set of $\Phi$ invariant states on $\mathscr{O}_{\mathbb{G}}(\Phi \backslash \mathbb{G})$ and the set of $\Phi$-bi-invariant states on $\mathscr{O}(\mathbb{G})$.

Proof. This is clear, because $\mathbb{E}_{\ell}^{\Phi}$ is completely positive.

Remark 3.6. We may note that given any functional $\nu: \mathscr{O}_{\mathbb{G}}(\Phi \backslash \mathbb{G}) \longrightarrow \mathbb{C}$ the functional $\mu: \mathscr{O}(\mathbb{G}) \longrightarrow \mathbb{C}$ defined by $\mu:=\nu \circ \mathbb{E}_{\ell}^{\Phi}$ is a left $\Phi$-invariant functional on $\mathscr{O}(\mathbb{G})$. This follows from the computations proving left $\Phi$-invariance of $\mu$ in the proof of Theorem 3.4 .

3.1.1. The case $\Phi=h_{\mathbb{K}} \circ \theta$ of quantum quotient spaces. Let $\mathbb{K}$ be a compact quantum subgroup of $\mathbb{G}$. Let $\theta$ be the associated surjective quantum group morphism. Then $\theta: \mathscr{O}(\mathbb{G}) \longrightarrow \mathscr{O}(\mathbb{K})$ is a surjective Hopf ${ }^{*}$-morphism such that $(\theta \otimes \theta) \circ \Delta=\Delta^{\prime} \circ \theta$ where $\Delta^{\prime}$ is the coproduct of $\mathscr{O}(\mathbb{K})$. It can be easily observed that $\beta_{r}:=($ id $\otimes \theta) \circ \Delta: \mathscr{O}(\mathbb{G}) \longrightarrow \mathscr{O}(\mathbb{G}) \otimes \mathscr{O}(\mathbb{K})$ is a right Hopf *-algebraic coaction of $\mathscr{O}(\mathbb{K})$ on the unital *-algebra $\mathscr{O}(\mathbb{G})$, and similarly $\beta_{l}:=(\theta \otimes \mathrm{id}) \circ \Delta: \mathscr{O}(\mathbb{G}) \longrightarrow \mathscr{O}(\mathbb{K}) \otimes \mathscr{O}(\mathbb{G})$ is a left Hopf *-algebraic coaction of $\mathscr{O}(\mathbb{K})$ on $\mathscr{O}(\mathbb{G})$.

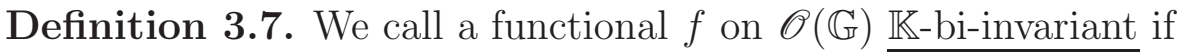

$$
(f \otimes \mathrm{id}) \circ \beta_{r}=f(\cdot) \mathbf{1}_{\mathbb{K}}=(\mathrm{id} \otimes f) \circ \beta_{l} .
$$


Let $h_{\mathbb{K}}$ be the Haar state of $\mathbb{K}$, so that $h:=h_{\mathbb{K}} \circ \theta \in \mathcal{I}(\mathbb{G})$.

Let $\operatorname{Irr}(\mathbb{K})$ be the set of inequivalent, irreducible unitary representations of $\mathbb{K}$. For $\pi \in \operatorname{Irr}(\mathbb{G})$, denote the carrier Hilbert space of $\pi$ by $H_{\pi}$, and let $\delta_{\pi}: H_{\pi} \longrightarrow H_{\pi} \otimes \mathscr{O}(\mathbb{K})$ be the $\mathscr{O}(\mathbb{K})$-comodule induced by $\pi$, as in DC16, Theorem 1.2, Lemma 1.7]. Then it follows that there exists an orthonormal basis $\left\{e_{1}, \ldots, e_{\operatorname{dim} \pi}\right\}$ of $H_{\pi}$ such that $\delta_{\pi}\left(e_{i}\right)=\sum_{k=1}^{\operatorname{dim} \pi} e_{k} \otimes \pi_{k j}$, cf. [DC16, Theorem 1.2, Lemma 1.5]. For $\pi \in \operatorname{Irr}(\mathbb{K})$, let $C(\mathbb{G})_{\pi}:=\operatorname{Lin}\left\{T \xi: \xi \in H_{\pi}, T \in \operatorname{Mor}\left(\pi, \beta_{r}\right)\right\}$, as in [DC16, Definition 3.13]. Then it follows from [DC16, Theorem 3.16] and its proof that $\mathscr{O}(\mathbb{G})=\bigoplus_{\pi \in \operatorname{Ir} r(\mathbb{K})} C(\mathbb{G})_{\pi}$.

Theorem 3.8. A functional $f$ on $\mathscr{O}(\mathbb{G})$ is left $\mathbb{K}$-invariant, i.e. $(f \otimes \mathrm{id}) \circ \beta_{r}=f$ if and only if for those $\pi \in \operatorname{Irr}(\mathbb{K})$ which are inequivalent to the trivial representation, we have $\left.f\right|_{C(\mathbb{G})_{\pi}}=0$.

Proof. Let $f$ be left $\mathbb{K}$-invariant. This implies that $(f \otimes \mathrm{id})\left(\beta_{r}(T \xi)\right)=f(T \xi)$ for all $\xi \in H_{\pi}$, for all $T \in \operatorname{Mor}\left(\pi, \beta_{r}\right)$. Thus in particular we have

$$
f\left(T\left(e_{j}\right)\right) \mathbf{1}_{\mathbb{K}}=(f \otimes \mathrm{id})\left(\beta_{r}\left(T\left(e_{j}\right)\right)\right)=(f \circ T \otimes \mathrm{id}) \delta_{\pi}\left(e_{j}\right),
$$

from which it follows that

$$
f\left(T\left(e_{j}\right)\right) \mathbf{1}_{\mathbb{K}}=\sum_{k=1}^{\operatorname{dim} \pi} f\left(T\left(e_{k}\right)\right) \pi_{k j}
$$

i.e.

$$
\sum_{k=1}^{\operatorname{dim} \pi} f\left(T\left(e_{k}\right)\right) \pi_{k j}-f\left(T\left(e_{j}\right)\right) \mathbf{1}_{\mathbb{K}}=0 .
$$

Since $\pi$ is different from the trivial representation, this means, using the linear independence of the set $\left\{e_{j}: j=1,2, \cdots \operatorname{dim} \pi\right\} \cup\left\{\mathbf{1}_{\mathbb{K}}\right\}, f\left(T\left(e_{j}\right)\right)=0$ for all $i, j$, which implies that $\left.f\right|_{C(\mathbb{G})_{\pi}}=0$.

Conversely suppose $\left.f\right|_{C(\mathbb{G}) \pi}=0$ for all those $\pi$ different from the trivial representation. Let $x \in \mathscr{O}(\mathbb{G})$. Since $\mathscr{O}(\mathbb{G})=\bigoplus_{\pi \in \operatorname{Irr}(\mathbb{K})} C(\mathbb{G})_{\pi}$ and $\beta_{r}\left(C(\mathbb{G})_{\pi}\right) \subset$ $C(\mathbb{G})_{\pi} \otimes \mathscr{O}(\mathbb{K})$, this implies that $(f \otimes$ id $) \beta_{r}(x)=0=f(x)$ if $x \in C(\mathbb{G})_{\pi}$ for $\pi \neq 11_{\mathbb{K}}$. If $x \in C(\mathbb{G})_{1_{\mathbb{K}}}$, then $x$ is a fixed point of the coaction $\beta_{r}$. Thus $(f \otimes \mathrm{id}) \beta_{r}(x)=f(x) \mathbf{1}_{\mathbb{K}}$. Thus $f$ is left $\mathbb{K}$-invariant.

It is now easy to also prove a corresponding right $\mathbb{K}$-invariance version of Theorem 3.8 :

Corollary 3.9. Let $f$ be a functional on $\mathscr{O}(\mathbb{G})$. Then $f$ is right $\mathbb{K}$-invariant i.e. $(\mathrm{id} \otimes f) \circ \beta_{l}=f$, if and only if $\left.f\right|_{C(\mathbb{G})_{\pi}}=0$ for all those $\pi \neq 1_{\mathbb{K}}$, where now for $\pi \in \operatorname{Irr}(\mathbb{K}), C(\mathbb{G})_{\pi}:=\left\{T \xi: \xi \in H_{\pi}, T \in \operatorname{Mor}\left(\pi, \beta_{l}\right)\right\}$.

We now prove the main results for this subsection. Note that $\Phi_{\mathbb{K}}=h_{\mathbb{K}} \circ \theta \in$ $\mathcal{I}(\mathbb{G})$. 
Theorem 3.10. A functional $f$ on $\mathscr{O}(\mathbb{G})$ is $\mathbb{K}$-bi-invariant if and only if it is $\Phi_{\mathbb{K}}$-bi-invariant.

Proof. If $f$ is $\mathbb{K}$-bi-invariant, it easily follows that $f$ is also $\Phi_{\mathbb{K}}$-bi-invariant.

We prove the converse implication. We prove only the left $\mathbb{K}$-invariance of $f$. The proof of the right $\mathbb{K}$-invariance is identical, with $\beta_{r}$ replaced by $\beta_{l}$.

We will use the notations in the proof of Theorem 3.8. Recall that

$$
\mathscr{O}(\mathbb{G})=\bigoplus_{\pi \in \operatorname{Irr}(\mathbb{K})} C(\mathbb{G})_{\pi}
$$

Let $x \in C(\mathbb{G})_{\pi}$, such that $\pi \neq \mathbf{1}_{\mathbb{K}}$. We may note that (id $\otimes h_{\mathbb{K}} \circ \theta$ ) $\circ \Delta$ is the conditioal expectation onto the fixed point subalgebra of the right coaction $\beta_{r}$. Since $\left(f \otimes h_{\mathbb{K}} \circ \theta\right)(\Delta(x))=f(x)$ and $\left(\mathrm{id} \otimes h_{\mathbb{K}} \circ \theta\right)(\Delta(x))=0$, this implies that $f(x)=0$. Left $\mathbb{K}$-invariance of $f$ now follows from Theorem 3.8 .

Let us recall the construction of the quantum quotient space $\mathbb{K} \backslash \mathbb{G}$ as explained in Subsection 1.3. As before, let us denote the Podleś algebra for the right action of $\mathbb{G}$ on $\mathbb{K} \backslash \mathbb{G}$ by $\mathscr{O}_{\mathbb{G}}(\mathbb{K} \backslash \mathbb{G})$, and the corresponding right Hopf ${ }^{*}$-coaction of $\mathscr{O}(\mathbb{G})$ on $\mathscr{O}_{\mathbb{G}}(\mathbb{K} \backslash \mathbb{G})$ by $\alpha$.

Definition 3.11. A functional $f$ on $\mathscr{O}_{\mathbb{G}}(\mathbb{K} \backslash \mathbb{G})$ is called $\mathbb{K}$-invariant if

$$
(\theta \otimes f) \circ \alpha=f(\cdot) \mathbf{1}_{\mathbb{K}} .
$$

Remark 3.12. We may note that the above definition of $\mathbb{K}$-invariance of a functional on $\mathscr{O}_{\mathbb{G}}(\mathbb{K} \backslash \mathbb{G}$ ) reduces to the usual definition of $K$-invariant measure on quotient spaces $K \backslash G$ when $G$ is a classical compact group and $K$ is a compact subgroup, as introduced in [L04, L15].

Let us also recall from Subsection 1.3 that $\mathscr{O}_{\mathbb{G}}(\mathbb{K} \backslash \mathbb{G}$ ) can equivalently be thought of as the right coidalgebra corresponding to the idempotent state $h_{\mathbb{K}} \circ \theta$ on $\mathscr{O}(\mathbb{G})$. Let $\mathbb{E}_{\mathbb{K} \backslash \mathbb{G}}:=\left(\mathrm{id} \otimes h_{\mathbb{K}} \circ \theta\right) \circ \Delta$ be the corresponding conditional expectation associated with the idempotent state $\Phi_{\mathbb{K}}=h_{\mathbb{K}} \circ \theta$.

Theorem 3.13. Let $f$ be a $\mathbb{K}$-invariant functional on $\mathscr{O}_{\mathbb{G}}(\mathbb{K} \backslash \mathbb{G})$. Then there exists a unique $\mathbb{K}$-bi-invariant functional $\mu$ on $\mathscr{O}(\mathbb{G})$ such that $f \circ \mathbb{E}_{\mathbb{K} \backslash \mathbb{G}}=\mu$.

Proof. Since $f$ is a $\mathbb{K}$-invariant functional on $\mathscr{O}_{\mathbb{G}}(\mathbb{K} \backslash \mathbb{G})$, this implies that $f$ is a $h_{\mathbb{K}} \circ \theta$-invariant functional in the sense of Definition 3.3 . Thus by Theorem 3.4, there exists a unique $h_{\mathbb{K}} \circ \theta$-bi-invariant functional $\mu$ on $\mathscr{O}(\mathbb{G})$ satisfying $f \circ \mathbb{E}_{\mathbb{K} \backslash \mathbb{G}}=\mu$. Now from Theorem 3.8 and Corollary 3.9 it follows that $\mu$ is also $\mathbb{K}$-bi-invariant as a functional on $\mathscr{O}(\mathbb{G})$. This proves the result.

As a consequence, we have the following:

Corollary 3.14. Let $\mu$ be a $\mathbb{K}$-bi-invariant functional on $\mathscr{O}(\mathbb{G})$. Then $f:=\left.\mu\right|_{\mathscr{O}_{\mathbb{G}}(\mathbb{K} \backslash \mathbb{G})}$ is the unique $\mathbb{K}$-invariant functional on $\mathscr{O}_{\mathbb{G}}\left(\mathbb{K} \backslash \mathbb{G}\right.$ ) such that $f \circ \mathbb{E}_{\mathbb{K} \backslash \mathbb{G}}=\mu$. 
Proof. We may note that the $\mathbb{K}$-invariance of the functional $f$ on $\mathscr{O}_{\mathbb{G}}(\mathbb{K} \backslash \mathbb{G})$ follows from the left $\mathbb{K}$-invariance of $\mu$ as a functional on $\mathscr{O}(\mathbb{G})$. The rest of the proof is an adaptation of the proof of Theorem 3.13 .

Thus we have a one-one correspondence between $\mathbb{K}$-invariant functionals on $\mathscr{O}_{\mathbb{G}}(\mathbb{K} \backslash \mathbb{G})$ and $\mathbb{K}$-bi-invariant functionals on $\mathscr{O}(\mathbb{G})$. This correspondence can be seen to extend the already known one-one correspondence between $K$-invariant measures on the quotient space $K \backslash G$ and $K$-bi-invariant measure on $G$ for a classical compact group $G$ and its compact subgroup $K$ [L04].

3.2. Convolution of functionals and invariant operators on expected right coidalgebras. Let $\mathscr{O}_{\mathbb{G}}(\Phi \backslash \mathbb{G})$ be an expected right coidalgebra and $h \in \mathcal{I}(\mathbb{G})$ be the associated idempotent state. Let $\varepsilon$ denote the counit of $\mathbb{G}$ and $\alpha:=\left.\Delta\right|_{\mathscr{O}_{\mathbb{G}}(\Phi \backslash \mathbb{G})}$.

\subsubsection{Convolution of functionals on expected right coidalgebras.}

Definition 3.15. Let $f$ and $g$ be two functionals on the expected right coidalgebra $\mathscr{O}_{\mathbb{G}}(\Phi \backslash \mathbb{G})$. We define convolution of $f$ and $g$, denoted $f \star_{\ell} g$ as the following functional on $\mathscr{O}_{\mathbb{G}}(\Phi \backslash \mathbb{G})$ :

$$
f \star_{\ell} g:=\left(f \otimes\left(g \circ \mathbb{E}_{\ell}^{\Phi}\right)\right) \circ \alpha .
$$

Remark 3.16. Let us make a remark on the notations used here:

For two functionals $\mu$ and $\nu$ on $\mathscr{O}(\mathbb{G}), \mu * \nu$ will denote the convolution defined by $\mu * \nu:=(\mu \otimes \nu) \circ \Delta$, whereas for two functionals $f$ and $g$ on $\mathscr{O}_{\mathbb{G}}(\Phi \backslash \mathbb{G}), f \star_{\ell} g$

will denote the functional on $\mathscr{O}_{\mathbb{G}}(\Phi \backslash \mathbb{G})$, as given in Definition 3.15.

Theorem 3.17. Let $f_{1}$ and $f_{2}$ be two $\Phi$-invariant functionals on $\mathscr{O}_{\mathbb{G}}(\Phi \backslash \mathbb{G})$ and $\mu_{1}$ and $\mu_{2}$ be their unique $\Phi$-bi-invariant extensions to $\mathscr{O}(\mathbb{G})$, as given by Theorem 3.4 .

Then the following holds:

(a) $f_{1} \star_{\ell} f_{2}$ is a $\Phi$-invariant functional on $\mathscr{O}_{\mathbb{G}}(\Phi \backslash \mathbb{G})$.

(b) $\mu_{1} * \mu_{2}$ is the unique $\Phi$-bi-invariant extension of $f_{1} \star_{\ell} f_{2}$ to $\mathscr{O}(\mathbb{G})$.

Proof. We prove (a):

$$
\begin{aligned}
\left(f_{1} \star_{\ell} f_{2} \otimes \Phi\right) \circ \alpha & =\left(f_{1} \otimes f_{2} \circ \mathbb{E}_{\ell}^{\Phi} \otimes \Phi\right) \circ(\alpha \otimes \mathrm{id}) \circ \alpha \\
& =\left(f_{1} \otimes f_{2} \circ \mathbb{E}_{\ell}^{\Phi} \otimes \Phi\right) \circ(\mathrm{id} \otimes \Delta) \circ \alpha \\
& =\left(f_{1} \otimes\left(f_{2} \circ \mathbb{E}_{\ell}^{\Phi} \otimes \Phi\right) \circ \Delta\right) \circ \alpha \\
& =\left(f_{1} \otimes\left(\mu_{2} \otimes \Phi\right) \circ \Delta\right) \circ \alpha \\
& =\left(f_{1} \otimes \mu_{2}\right) \circ \alpha \quad\left(\text { using left } \Phi \text {-invariance of } \mu_{2}\right) \\
& =\left(f_{1} \otimes f_{2} \circ \mathbb{E}_{\ell}^{\Phi}\right) \circ \alpha=f_{1} \star_{\ell} f_{2} .
\end{aligned}
$$

To prove (b): 
Using the fact that both $\mu_{1}$ and $\mu_{2}$ are $\Phi$-bi-invariant functionals on $\mathscr{O}(\mathbb{G})$, it is easy to see that $\mu_{1} * \mu_{2}$ is a $\Phi$-bi-invariant functional on $\mathscr{O}(\mathbb{G})$.

Let $x \in \mathscr{O}_{\mathbb{G}}(\Phi \backslash \mathbb{G})$.

$$
\begin{aligned}
\left(\mu_{1} * \mu_{2}\right)(x) & =\left(\mu_{1} * \mu_{2}\right)\left(\mathbb{E}_{\ell}^{\Phi}(x)\right) \\
& =\left(f_{1} \circ \mathbb{E}_{\ell}^{\Phi} \otimes f_{2} \circ \mathbb{E}_{\ell}^{\Phi}\right)\left(\Delta\left(\mathbb{E}_{\ell}^{\Phi}(x)\right)\right) \\
& \left.=\left(f_{1} \circ\left(\mathbb{E}_{\ell}^{\Phi}\right)^{2} \otimes f_{2} \circ \mathbb{E}_{\ell}^{\Phi}\right)(\Delta(x)) \quad \text { (using the invariance of } \mathbb{E}_{\ell}^{\Phi}\right) \\
& =\left(f_{1} \circ \mathbb{E}_{\ell}^{\Phi} \otimes f_{2} \circ \mathbb{E}_{\ell}^{\Phi}\right)(\Delta(x)) \\
& =\left(f_{1} \otimes f_{2} \circ \mathbb{E}_{\ell}^{\Phi}\right)\left(\Delta\left(\mathbb{E}_{\ell}^{\Phi}(x)\right)\right) \\
& \left.=\left(f_{1} \otimes f_{2} \circ \mathbb{E}_{\ell}^{\Phi}\right)(\alpha(x))\right)=f_{1} \star_{\ell} f_{2}(x)
\end{aligned}
$$

which proves that $\left.\mu_{1} * \mu_{2}\right|_{\mathscr{O}_{\mathbb{G}}(\Phi \backslash \mathbb{G})}=f_{1} \star_{\ell} f_{2}$. It now follows from Theorem 3.4 that $\mu_{1} * \mu_{2}$ must be the unique $\Phi$-bi-invariant extension of $f_{1} \star_{\ell} f_{2}$ to $\mathscr{O}(\mathbb{G})$.

3.2.2. $\mathbb{G}$-invariant operators on expected right coidalgebras. Recall that linear map $T: \mathscr{O}_{\mathbb{G}}(\Phi \backslash \mathbb{G}) \longrightarrow \mathscr{O}_{\mathbb{G}}(\Phi \backslash \mathbb{G})$ is called $\mathbb{G}$-invariant if $(T \otimes \mathrm{id}) \circ \alpha=\alpha \circ T$, see Definition 2.2.

Remark 3.18. This definition is motivated by the following observation:

If $G$ is a classical compact group, then all expected right coidalgebras of $(C(G), \Delta)$, where $\Delta$ is the canonical coproduct on $C(G)$, are of the form $C(K \backslash G)$, for some compact subgroup $K \subset G$.

A linear map $T: C(K \backslash G) \longrightarrow C(K \backslash G)$ is called $G$-invariant, if $T$ is covariant with respect to the canonical action of $G$ on $C(K \backslash G)$ [L04, L15], i.e. denoting the action of $G$ on $C(K \backslash G)$ by $G \ni g \mapsto \lambda_{g} \in A u t(C(K \backslash G))$, we have $T \circ \lambda_{g}=\lambda_{g} \circ T$ for all $g \in G$.

Let $E_{\pi}(\cdot):=\int_{G} \chi_{\pi}(g) \lambda_{g}(\cdot) d g$, where $d g$ is the Haar measure of $G, \pi$ is an irreducible unitary representation of $G$ and $\chi_{\pi}$ is its character. It can be seen that $E_{\pi}$ is a completely bounded idempotent and $C(K \backslash G)_{\pi}:=\left\{E_{\pi}(f): f \in\right.$ $C(K \backslash G)\}$ is the spectral subspace of $C(K \backslash G)$ for the action $\lambda$, corresponding to $\pi$. Denoting $\mathscr{O}_{G}(K \backslash G):=\bigoplus_{\pi} C(K \backslash G)_{\pi}$ and $\lambda: C(K \backslash G) \longrightarrow C(K \backslash G) \otimes C(G)$ by $\lambda(f)(x, g):=\lambda_{g}(f)(x)$, it follows that $\left.\lambda\right|_{\mathscr{O}_{G}(K \backslash G)}: \mathscr{O}_{G}(K \backslash G) \longrightarrow \mathscr{O}_{G}(K \backslash G) \otimes$ $\mathscr{O}_{G}(G)$ is a right coaction of the Hopf ${ }^{*}$-algebra $\left(\mathscr{O}_{G}(G), \Delta\right)$, where $\Delta$ is the restriction of the canonical coproduct on $C(G)$.

Using the covariance of $T$, it is possible to see now that $T\left(\mathscr{O}_{G}(K \backslash G)\right) \subset \mathscr{O}_{G}(K \backslash G)$ and $\left.(T \otimes \mathrm{id}) \circ \lambda\right|_{\mathscr{O}_{G}(K \backslash G)}=\left.\lambda\right|_{\mathscr{O}_{G}(K \backslash G)} \circ T$.

Lemma 3.19. Let $T: \mathscr{O}_{\mathbb{G}}(\Phi \backslash \mathbb{G}) \longrightarrow \mathscr{O}_{\mathbb{G}}(\Phi \backslash \mathbb{G})$ be $\mathbb{G}$-invariant. Then $\gamma:=\varepsilon \circ T$ is a $\Phi$-invariant functional on $\mathscr{O}_{\mathbb{G}}(\Phi \backslash \mathbb{G})$.

Conversely, if $\gamma$ is a functional on $\mathscr{O}_{\mathbb{G}}(\Phi \backslash \mathbb{G})$, then the formula $T:=\left(\gamma \otimes \mathbb{E}_{\ell}^{\Phi}\right) \circ \alpha$ defines a $\mathbb{G}$-invariant map on $\mathscr{O}_{\mathbb{G}}(\Phi \backslash \mathbb{G})$. However, $\gamma=\varepsilon \circ T$ if and only if $\gamma$ is $\Phi$-invariant. 
Proof. The $\Phi$-invariance of $\gamma$ can be seen as follows:

$$
\begin{aligned}
(\gamma \otimes \Phi)(\alpha(x)) & =(\varepsilon \circ T \otimes \Phi)(\alpha(x)) \\
& =(\varepsilon \otimes \Phi)(\alpha(T x)) \\
& =\Phi(T x) \\
& =\varepsilon\left(\mathbb{E}_{\ell}^{\Phi}(T x)\right) \\
& =\varepsilon(T x)=\gamma(x) \quad\left(\text { as } T x \in \mathscr{O}_{\mathbb{G}}(\Phi \backslash \mathbb{G})\right) .
\end{aligned}
$$

Now let $\gamma: \mathscr{O}_{\mathbb{G}}(\Phi \backslash \mathbb{G}) \longrightarrow \mathbb{C}$ be a functional. Then

$$
\begin{aligned}
(T \otimes \mathrm{id})(\alpha(x)) & =\left(\gamma \otimes \mathbb{E}_{\ell}^{\Phi} \otimes \mathrm{id}\right)(\alpha \otimes \mathrm{id})(\alpha(x)) \\
& =\left(\gamma \otimes \mathbb{E}_{\ell}^{\Phi} \otimes \mathrm{id}\right)(\mathrm{id} \otimes \Delta)(\alpha(x)) \\
& =\left\{\gamma \otimes\left(\mathbb{E}_{\ell}^{\Phi} \otimes \mathrm{id}\right) \circ \Delta\right\}(\alpha(x)) \\
& =\left(\gamma \otimes \Delta \circ \mathbb{E}_{\ell}^{\Phi}\right)(\alpha(x)) \\
& =\Delta\left(\left(\gamma \otimes \mathbb{E}_{\ell}^{\Phi}\right)(\alpha(x))\right)=\Delta(T x),
\end{aligned}
$$

which proves the $\mathbb{G}$-invariance of $T$.

We may now observe that

$$
\left.\varepsilon(T x)=\left(\gamma \otimes \varepsilon \circ \mathbb{E}_{\ell}^{\Phi}\right)(\alpha)(x)\right)=(\gamma \otimes h)(\alpha(x)),
$$

from which it follows that $\varepsilon \circ T=\gamma$ if and only if $\gamma$ is $\Phi$-invariant.

The above lemma leads to the following observation:

Theorem 3.20. There exists a one-to-one correspondence between $\Phi$-invariant functionals on $\mathscr{O}_{\mathbb{G}}(\Phi \backslash \mathbb{G})$ (denoted by $\gamma$ ) and $\mathbb{G}$-invariant operators on $\mathscr{O}_{\mathbb{G}}(\Phi \backslash \mathbb{G}$ ) (denoted by $T)$, given by

$$
\begin{gathered}
\gamma \mapsto T:=\left(\gamma \otimes \mathbb{E}_{\ell}^{\Phi}\right) \circ \alpha ; \\
T \mapsto \gamma:=\varepsilon \circ T .
\end{gathered}
$$

We now relate the convolution of $\Phi$-invariant functionals on $\mathscr{O}_{\mathbb{G}}(\Phi \backslash \mathbb{G})$ with composition of $\mathbb{G}$-invariant operators on $\mathscr{O}_{\mathbb{G}}(\Phi \backslash \mathbb{G})$.

Theorem 3.21. Let $\gamma_{1}$ and $\gamma_{2}$ be two $\Phi$-invariant functionals on $\mathscr{O}_{\mathbb{G}}(\Phi \backslash \mathbb{G})$, and $T_{1}$ and $T_{2}$ be the corresponding $\mathbb{G}$-invariant operators (or vice-versa as given by Theorem 3.20). Then we have

$$
\gamma_{1} \star_{\ell} \gamma_{2}=\varepsilon \circ T_{2} \circ T_{1} .
$$


Proof. For $x \in \mathscr{O}_{\mathbb{G}}(\Phi \backslash \mathbb{G})$ observe that

$$
\begin{aligned}
\left(\gamma_{1} \star_{\ell} \gamma_{2}\right)(x) & =\left(\gamma_{1} \otimes \gamma_{2} \circ \mathbb{E}_{\ell}^{\Phi}\right)(\alpha(x)) \\
& =\left(\varepsilon \circ T_{1} \otimes \varepsilon \circ T_{2} \circ E_{1}\right)(\alpha(x)) \\
& =\left(\varepsilon \otimes \varepsilon \circ T_{2} \circ \mathbb{E}_{\ell}^{\Phi}\right)\left(\alpha\left(T_{1} x\right)\right) \\
& =\varepsilon\left(T_{2}\left(\mathbb{E}_{\ell}^{\Phi}\left(T_{1} x\right)\right)\right) \\
& =\varepsilon\left(T_{2}\left(T_{1}(x)\right)\right),
\end{aligned}
$$

which proves our claim.

\section{MARKOV SEMIGROUPS ON EXPECTED RIGHT COIDALGEBRAS}

As before, we fix $\Phi \in \mathcal{I}(\mathbb{G})$ and let $\mathbb{E}_{\ell}^{\Phi}:=(\Phi \otimes$ id $) \circ \Delta, \mathbb{E}_{r}^{\Phi}:=($ id $\otimes \Phi) \circ \Delta$, $\mathscr{O}_{\mathbb{G}}(\Phi \backslash \mathbb{G}):=\mathbb{E}_{\ell}^{\Phi}(\mathscr{O}(\mathbb{G}))$ and $\mathscr{O}_{\mathbb{G}}(\mathbb{G} / \Phi):=\mathbb{E}_{r}^{\Phi}(\mathscr{O}(\mathbb{G}))$.

A one parameter family of (G-Ginvariant) operators $T:=\left\{T_{t}: \mathscr{O}_{\mathbb{G}}(\Phi \backslash \mathbb{G}) \longrightarrow\right.$ $\left.\mathscr{O}_{\mathbb{G}}(\Phi \backslash \mathbb{G})\right\}_{t \geq 0}$ will be called a semigroup of operators if $T_{t+s}=T_{t} \circ T_{s}$.

4.1. Structure of convolution semigroups of invariant functionals on expected right coidalgebras. The convolution on $\mathscr{O}_{\mathbb{G}}(\mathbb{K} \backslash \mathbb{G}$ ) allows us to define convolution semigroups of functionals or states on $\mathscr{O}_{\mathbb{G}}(\mathbb{K} \backslash \mathbb{G})$ in the same way as in Definition 1.4.

Definition 4.1. A convolution semigroup on an expected rigth coidalgebra $\mathscr{O}_{\mathbb{G}}(\Phi \backslash \mathbb{G})$ is a family of linear functionals $\left(\lambda_{t}: \mathscr{O}_{\mathbb{G}}(\Phi \backslash \mathbb{G}) \rightarrow \mathbb{C}\right)_{t \geq 0}$ such that

(1) $\lim _{t \searrow_{0}} \lambda_{t}(a)=\lambda_{0}(a)$ for all $a \in \mathscr{O}_{\mathbb{G}}(\mathbb{K} \backslash \mathbb{G}$ ) (weak continuity);

(2) $\lambda_{s} \star_{\ell} \lambda_{t}=\lambda_{s+t}$ for all $s, t \geq 0$ (semigroup property).

We call $\left(\lambda_{t}\right)_{t \geq 0}$ a convolution semigroup of states, if the functionals $\lambda_{t}$ are furthermore normalized, i.e., $\lambda_{t}(1)=1$, and positive, i.e., $\lambda_{t}\left(a^{*} a\right) \geq 0$ for all $a \in \mathscr{O}_{\mathbb{G}}(\Phi \backslash \mathbb{G})$ and all $t \geq 0$.

Lemma 4.2. Let $\lambda:=\left\{\lambda_{t}: \mathscr{O}_{\mathbb{G}}(\Phi \backslash \mathbb{G}) \longrightarrow \mathbb{C}\right\}_{t \geq 0}$ be a convolution semigroup of $\Phi$-invariant functionals on $\mathscr{O}_{\mathbb{G}}(\Phi \backslash \mathbb{G})$. For each $t \geq 0$ let $\mu_{t}: \mathscr{O}(\mathbb{G}) \longrightarrow \mathbb{C}$ be the unique $\Phi$-bi-invariant extension of $\lambda_{t}$, as given by Theorem 3.4. Then $\mu:=\left\{\mu_{t}: \mathscr{O}(\mathbb{G}) \longrightarrow \mathbb{C}\right\}_{t \geq 0}$ is a convolution semigroup of functionals on $\mathscr{O}(\mathbb{G})$.

Proof. Fix $t, s \in[0,+\infty)$. It follows that

$$
\left.\left(\mu_{t} * \mu_{s}\right)\right|_{\mathscr{O}_{\mathbb{G}}(\Phi \backslash \mathbb{G})}=\lambda_{t} \star_{\ell} \lambda_{s}=\lambda_{t+s}=\left.\mu_{t+s}\right|_{\mathscr{O}_{\mathbb{G}}(\Phi \backslash \mathbb{G})},
$$

where the first equality follows from Theorem 3.17. (b). By the same, we know that $\mu_{t} * \mu_{s}$ is the unique $\Phi$-bi-invariant extension of $\lambda_{t} \star_{\ell} \lambda_{s}=\lambda_{t+s}$. This implies that $\mu_{t} * \mu_{s}=\mu_{t+s}$.

Weak continuity easily follows from the formula $\mu_{t}=\lambda_{t} \circ \mathbb{E}_{\ell}^{\Phi}$.

Remark 4.3. In general the convolution semigroup $\left(\mu_{t}\right)_{t \geq 0}$ does not start with the counit. Instead we have $\mu_{0}=\lambda_{0} \circ \mathbb{E}_{\ell}^{\Phi}=\Phi * \lambda_{0}$. 
We next prove an automatic $\Phi$-invariance of convolution semigroup of functionals, starting at a state.

Lemma 4.4. Let $\left\{\lambda_{t}: \mathscr{O}_{\mathbb{G}}(\Phi \backslash \mathbb{G}) \longrightarrow \mathbb{C}\right\}_{t \geq 0}$ be a convolution semigroup of functionals such that $\lambda_{0}$ is a state on $\left(\mathscr{O}_{\mathbb{G}}(\Phi \backslash \mathbb{G})\right.$, i.e., $\lambda_{0}\left(x^{*} x\right) \geq 0$ and $\lambda_{0}(\nVdash)=1$. Then for each $t \geq 0, \lambda_{t}$ is $\Phi$-invariant.

Proof. Let $\mu_{t}:=\lambda_{t} \circ \mathbb{E}_{\ell}^{\Phi}$. Lemma 4.2 implies that $\left(\mu_{t}\right)_{t \geq 0}$ is a convolution semigroup of functionals on $\mathscr{O}(\mathbb{G})$, such that for each $t \geq 0, \mu_{t}$ is a left $\Phi$-invariant functional on $\mathscr{O}(\mathbb{G})$. Let us first show that $\mu_{t}$ is $\Phi$-bi-invariant.

We may note that $\mu_{0}:=\lambda_{0} \circ \mathbb{E}_{\ell}^{\Phi}$ is an idempotent state on $\mathscr{O}(\mathbb{G})$. Moreover, as $\mu_{t}$ is left $\Phi$-invariant for each $t \geq 0$, this implies in particular that $\Phi * \mu_{0}=\mu_{0}$. Hence by Lemma [3.2, we have $\mu_{0} * \Phi=\mu_{0}$. This implies that $\mu_{t} *\left(\mu_{0} * \Phi\right)=\mu_{t}$ i.e. $\mu_{t} * \Phi=\mu_{t}$ for all $t \geq 0$. Hence $\left(\mu_{t}\right)_{t \geq 0}$ is a convolution semigroup of $\Phi$-biinvariant functionals on $\mathscr{O}(\mathbb{G})$. Theorem 3.4 now yields that $\lambda_{t}=\left.\mu_{t}\right|_{\mathscr{O}_{\mathbb{G}}(\Phi \backslash \mathbb{G})}$ must be $\Phi$-invariant for each $t \geq 0$. This proves the claim.

We will now have a look at the differentiability properties of convolution semigroups on $\mathscr{O}_{\mathbb{G}}(\Phi \backslash \mathbb{G})$ and the associated operator semigroups.

Proposition 4.5. Let $\left(\lambda_{t}\right)_{t \geq 0}$ be a pointwise continuous convolution semigroup of $\Phi$-invariant functionals on $\mathscr{O}_{\mathbb{G}}(\Phi \backslash \mathbb{G})$. Then for each $x \in \mathscr{O}_{\mathbb{G}}(\Phi \backslash \mathbb{G})$, the function $[0,+\infty) \ni t \mapsto \lambda_{t}(x) \in \mathbb{C}$ is differentiable at $t=0$.

Proof. Let $\left(\mu_{t}\right)_{t \geq 0}$ be the unique $\Phi$-bi-invariant extension of $\left(\lambda_{t}\right)_{t \geq 0}$. This is a continuous convolution semigroup of linear functionals and the discussion in [FS00, Section 3] shows that it is differentiable, which implies the differentiability of $\left(\lambda_{t}\right)_{t \geq 0}$.

The following result is an 'operator' version of Proposition 4.5.

Proposition 4.6. Let $\left\{T_{t}: \mathscr{O}_{\mathbb{G}}(\Phi \backslash \mathbb{G}) \longrightarrow \mathscr{O}_{\mathbb{G}}(\Phi \backslash \mathbb{G})\right\}_{t \geq 0}$ be a poinwise continuous (w.r.t. to the universal $C^{*}$-norm) one parameter semigroup such that for each $t \geq 0, T_{t}$ is $\mathbb{G}$-invariant. Then for each $x \in \mathscr{O}_{\mathbb{G}}(\Phi \backslash \mathbb{G})$, the map $[0,+\infty) \ni t \mapsto$ $T_{t}(x) \in \mathscr{O}_{\mathbb{G}}(\Phi \backslash \mathbb{G})$ is differentiable at 0 .

Proof. This follows by applying Proposition 4.5 to $\lambda_{t}=\varepsilon \circ T_{t}$ and using Theorem 3.20 .

The next result is a converse of Proposition 4.5.

Proposition 4.7. Let $\psi: \mathscr{O}_{\mathbb{G}}(\Phi \backslash \mathbb{G}) \longrightarrow \mathbb{C}$ be a $\Phi$-invariant map. Then there exists a strongly continuous convolution semigroup $\left\{\lambda_{t}: \mathscr{O}_{\mathbb{G}}(\Phi \backslash \mathbb{G}) \longrightarrow \mathbb{C}\right\}_{t \geq 0}$ consisting of $\Phi$-invariant maps and $\lambda_{0}=\left.\varepsilon\right|_{\mathscr{O}_{\mathbb{G}}(\Phi \backslash \mathbb{G})}$, such that $\psi=\left.\frac{d}{d t}\right|_{t=0} \lambda_{t}$.

Proof. For $x \in \mathscr{O}_{\mathbb{G}}(\Phi \backslash \mathbb{G})$, define $A(x):=\left(\psi \otimes \mathbb{E}_{\ell}^{\Phi}\right)(\Delta(x))$. From Theorem 3.20 , it follows that $A: \mathscr{O}_{\mathbb{G}}(\Phi \backslash \mathbb{G}) \longrightarrow \mathscr{O}_{\mathbb{G}}(\Phi \backslash \mathbb{G})$ is a $\mathbb{G}$-invariant operator. 
Fix $x \in \mathscr{O}_{\mathbb{G}}(\Phi \backslash \mathbb{G})$. We can use fundamental theorem of coalgebras to restrict to finite-dimensional subcoalgebra $X$ that contains $x$, one sees that

$$
T_{t}(x):=\sum_{k=0}^{\infty} \frac{t^{k}}{k !} A^{k}(x)
$$

converges on $X$. Since $x$ was arbitrary, the convergence holds for all $x \in \mathscr{O}_{\mathbb{G}}(\Phi \backslash \mathbb{G})$ and defines a semigroup of $\mathbb{G}$-invariant operators.

Let $\lambda_{t}:=\varepsilon \circ T_{t}$ for each $t$. An application of Theorem 3.20 and Lemma 4.2 implies that $\left(\lambda_{t}\right)_{t \geq 0}$ is a convolution semigroup of $\Phi$-invariant functionals on $\mathscr{O}_{\mathbb{G}}(\Phi \backslash \mathbb{G})$. Since $X$ is finite dimensional, $\left.\varepsilon\right|_{X}$ is a bounded functional on $X$. From this, it follows easily that the map $[0,+\infty) \ni t \mapsto \lambda_{t}(x)$ is continuous at 0 . The result follows now.

Corollary 4.8. Let $\Phi \in \mathcal{I}(\mathbb{G})$ and $\psi: \mathscr{O}(\mathbb{G}) \longrightarrow \mathbb{C}$ be a functional which is $\Phi$-bi-invariant. Then there exists a convolution semigroup of functionals $\left\{\lambda_{t}\right.$ : $\mathscr{O}(\mathbb{G}) \longrightarrow \mathbb{C}\}_{t \geq 0}$ such that for each $t \geq 0, \lambda_{t}$ is $\Phi$-bi-invariant, and $\lambda_{0}=\Phi$ and $\left.\frac{d}{d t}\right|_{t=0} \lambda_{t}=\psi$.

Proof. Let $\mathbb{E}_{\ell}^{\Phi}:=(\Phi \otimes \mathrm{id}) \circ \Delta$ and $\mathscr{O}_{\mathbb{G}}(\Phi \backslash \mathbb{G}):=\mathbb{E}_{\ell}^{\Phi}(\mathscr{O}(\mathbb{G}))$. Then $\phi:=\left.\psi\right|_{\mathscr{O}_{\mathbb{G}}(\Phi \backslash \mathbb{G})}$ is a $\Phi$-invariant functional on the expected right coidalgebra $\mathscr{O}_{\mathbb{G}}(\Phi \backslash \mathbb{G})$. Then by Theorem 4.7, it follows that there exists a convolution semigroup $\left\{\beta_{t}: \mathscr{O}_{\mathbb{G}}(\Phi \backslash \mathbb{G}) \longrightarrow\right.$ $\mathbb{C}\}_{t \geq 0}$ such that for each $t \geq 0, \beta_{t}$ is a $\Phi$-bi-invariant functional on $\mathscr{O}_{\mathbb{G}}(\Phi \backslash \mathbb{G})$, $\beta_{0}=\left.\varepsilon\right|_{\mathscr{O}_{\mathbb{G}}(\Phi \backslash \mathbb{G})}$ and $\left.\frac{d}{d t}\right|_{t=0} \beta_{t}=\phi$. Let $\left\{\lambda_{t}: \mathscr{O}(\mathbb{G}) \longrightarrow \mathbb{C}\right\}_{t \geq 0}$ be the extension of $\left(\beta_{t}\right)_{t \geq 0}$ to a $\Phi$-bi-invariant convolution semigroup of functionals on $\mathscr{O}(\mathbb{G})$, as given by Lemma 4.2. It now follows that $\left(\lambda_{t}\right)_{t \geq 0}$ is the required convolution semigroup with the desired property.

\subsection{Structure of convolution semigroups of states on expected coidal- gebras.}

Remark 4.9. It is worthwhile to note at this point that Theorem 3.20 along with Lemma ?? essentially gives us a way to go back and forth between convolution semigroup of states on $\mathscr{O}_{\mathbb{G}}(\Phi \backslash \mathbb{G})$ and $\mathbb{G}$-invariant Markov semigroup on $\mathscr{O}_{\mathbb{G}}(\Phi \backslash \mathbb{G})$.

The following theorem gives a Schoenberg correspondence for expected right coidalgebras.

Theorem 4.10. Let $\left\{\lambda_{t}: \mathscr{O}_{\mathbb{G}}(\Phi \backslash \mathbb{G}) \longrightarrow \mathbb{C}\right\}_{t \geq 0}$ be a strongly continous convolution semigroup of functionals. Let $\psi:=\left.\frac{\bar{d}}{d t} \lambda_{t}\right|_{t=0}$. Then the following are equivalent.

(i) $\left(\lambda_{t}\right)_{t \geq 0}$ is a convolution semigroup of states.

(ii) $\psi$ is a well-defined map on $\mathscr{O}_{\mathbb{G}}(\Phi \backslash \mathbb{G}), \lambda_{0}$ is positive and $\psi\left(x^{*} x\right) \geq 0$ for all $x \in \mathscr{O}_{\mathbb{G}}(\Phi \backslash \mathbb{G})$ with $\lambda_{0}\left(x^{*} x\right)=0$, and $\psi\left(x^{*}\right)=\overline{\psi(x)}$ for all $x \in \mathscr{O}_{\mathbb{G}}(\Phi \backslash \mathbb{G})$. 
Proof. Let us first extend $\left(\lambda_{t}\right)_{t \geq 0}$ to a convolution semigroup $\left\{\mu_{t}: \mathscr{O}(\mathbb{G}) \longrightarrow \mathbb{C}\right\}_{t \geq 0}$ of $\Phi$-bi-invariant functionals, as shown in Lemma 4.4. As in the proof of Theorem 4.5, $\left(\mu_{t}\right)_{t \geq 0}$ is also strongly continuous. Moreover, since for each $t \in[0,+\infty)$, $\mu_{t}=\lambda_{t} \circ \mathbb{E}_{\ell}^{\Phi}$, this implies that $\mu_{t}$ is a state on $\mathscr{O}(\mathbb{G})$ for all $t$. Moreover, from the proof of Theorem 4.5 it follows that $\psi \circ \mathbb{E}_{\ell}^{\Phi}=\left.\frac{d}{d t} \mu_{t}\right|_{t=0}$. So it is enough to prove (i) and (ii) for $\left(\mu_{t}\right)_{t \geq 0}$. Since $(\mathscr{O}(\mathbb{G}), \Delta)$ is a ${ }^{*}$-bialgebra, the result now follows from [FS00, Theorem 3.3].

\section{Quantum hypergroups}

5.1. Functionals on the algebra of $\Phi$-bi-invariant functions on $\mathbb{G}$. Let $\Phi \in \mathcal{I}(\mathbb{G})$ and denote $\mathbb{E}_{\ell}^{\Phi}:=(\Phi \otimes \mathrm{id}) \circ \Delta$ and $\mathbb{E}_{r}^{\Phi}:=(\operatorname{id} \otimes \Phi) \circ \Delta$. Let $\mathscr{O}_{\mathbb{G}}(\Phi \backslash \mathbb{G}):=$ $\mathbb{E}_{\ell}^{\Phi}(\mathscr{O}(\mathbb{G}))$ and $\mathscr{O}_{\mathbb{G}}(\mathbb{G} / \Phi):=\mathbb{E}_{r}^{\Phi}(\mathscr{O}(\mathbb{G}))$.

Definition 5.1. The *-algebra of $\Phi$-bi-invariant functions on $\mathbb{G}$, denoted by $\mathscr{O}_{\mathbb{G}}(\Phi \backslash \mathbb{G} / \Phi)$ is defined by $\mathscr{O}_{\mathbb{G}}(\Phi \backslash \mathbb{G} / \Phi):=\mathscr{O}_{\mathbb{G}}(\Phi \backslash \mathbb{G}) \cap \mathscr{O}_{\mathbb{G}}(\mathbb{G} / \Phi)=\mathbb{E}_{\ell}^{\Phi}\left(\mathbb{E}_{r}^{\Phi}(\mathscr{O}(\mathbb{G}))\right)$.

Remark 5.2. It is worthwhile to note that in the context of CQG algebras, the double coset hyper bialgebra considered in [FS00 is a special case of the algebra introduced in Definition [5.1. Haonan Zhang [Zh18, Proposition 2.4] has shown that $C(\Phi \backslash \mathbb{G} / \Phi)=\mathbb{E}_{\ell}^{\Phi}\left(\mathbb{E}_{r}^{\Phi}(C(\mathbb{G}))\right)$ has the structure of a compact quantum hypergroup in the sense of [ChV99.

Theorem 5.3. Let $f$ be a functional on $\mathscr{O}_{\mathbb{G}}(\Phi \backslash \mathbb{G} / \Phi)$ and define $\mu:=f \circ \mathbb{E}_{\ell}^{\Phi} \circ \mathbb{E}_{r}^{\Phi}$. Then $\mu$ is the unique $\Phi$-bi-invariant functional on $\mathscr{O}(\mathbb{G})$ such that $\left.\mu\right|_{\mathscr{O}_{\mathbb{G}}(\Phi \backslash \mathbb{G} / \Phi)}=f$.

Proof. We prove the $\Phi$-bi-invariance of $\mu$ as a functional on $\mathscr{O}(\mathbb{G})$. We may note that an easy computation yields $\mathbb{E}_{r}^{\Phi} \circ \mathbb{E}_{\ell}^{\Phi}=\mathbb{E}_{\ell}^{\Phi} \circ \mathbb{E}_{r}^{\Phi}$. We only show the left $\Phi$-invariance of $\mu$. The proof of right $\Phi$-invariance is identical. Let $x \in \mathscr{O}(\mathbb{G})$.

$$
\begin{aligned}
(\Phi \otimes \mu)(\Delta(x)) & =\left(\Phi \otimes f \circ \mathbb{E}_{\ell}^{\Phi} \circ \mathbb{E}_{r}^{\Phi}\right)(\Delta(x)) \\
& =f\left(\mathbb{E}_{\ell}^{\Phi}\left(\mathbb{E}_{r}^{\Phi}\left(\mathbb{E}_{\ell}^{\Phi}(x)\right)\right)\right) \\
& =f\left(\mathbb{E}_{r}^{\Phi}\left(\left(\mathbb{E}_{\ell}^{\Phi}\right)^{2}(x)\right)\right) \\
& =f\left(\mathbb{E}_{r}^{\Phi}\left(\mathbb{E}_{\ell}^{\Phi}(x)\right)\right) \\
& =f\left(\mathbb{E}_{\ell}^{\Phi}\left(\mathbb{E}_{r}^{\Phi}(x)\right)\right)=\mu(x),
\end{aligned}
$$

which proves left $\Phi$-invariance of $\mu$.

Conversely, suppose $\nu$ is a $\Phi$-invariant functional on $\mathscr{O}(\mathbb{G})$ such that $\left.\nu\right|_{\mathscr{O}_{\mathbb{G}}(\Phi \backslash \mathbb{G} / \Phi)}=$ $f$. Let $x \in \mathscr{O}(\mathbb{G})$. We have

$$
\begin{aligned}
\nu(x) & =(\Phi \otimes \nu)(\Delta(x)) \\
& =\nu\left(\mathbb{E}_{\ell}^{\Phi}(x)\right) \\
& =(\nu \otimes \Phi)\left(\Delta\left(\mathbb{E}_{\ell}^{\Phi}(x)\right)\right) \\
& =\nu\left(\mathbb{E}_{r}^{\Phi}\left(\mathbb{E}_{\ell}^{\Phi}(x)\right)\right) \\
& =f\left(\mathbb{E}_{\ell}^{\Phi}\left(\mathbb{E}_{r}^{\Phi}(x)\right)\right)=\mu(x),
\end{aligned}
$$


which proves the uniqueness.

A functional on $f$ on $\mathscr{O}_{\mathbb{G}}(\Phi \backslash \mathbb{G} / \Phi)$ can be extended in many ways to a functional on the right coidalgebra $\mathscr{O}_{\mathbb{G}}(\Phi \backslash \mathbb{G})$. For example, let $x \in \mathscr{O}_{\mathbb{G}}(\Phi \backslash \mathbb{G})$. Then $x$ admits a unique decomposition $x=a+b$, where $a \in \mathbb{E}_{r}^{\Phi}(\mathscr{O}(\mathbb{G}))$ and $b \in\left(\mathbb{E}_{r}^{\Phi}\right)^{\perp}(\mathscr{O}(\mathbb{G}))$. Note that $a \in \mathscr{O}_{\mathbb{G}}(\Phi \backslash \mathbb{G} / \Phi)$. Now the assignment $x \mapsto f(a)+\psi(b)$, for any functional $\psi$ on $\left(\mathbb{E}_{r}^{\Phi}\right)^{\perp}(\mathscr{O}(\mathbb{G}))$, gives a well-defined functional on $\mathscr{O}_{\mathbb{G}}(\Phi \backslash \mathbb{G})$. However, not all such extensions will be $\Phi$-invariant as functionals on $\mathscr{O}_{\mathbb{G}}(\Phi \backslash \mathbb{G})$. In fact we have

Corollary 5.4. Let $f$ be a functional on $\mathscr{O}_{\mathbb{G}}(\Phi \backslash \mathbb{G} / \Phi)$. Then there exists a unique functional $\lambda$ on $\mathscr{O}_{\mathbb{G}}(\Phi \backslash \mathbb{G})$ such that

- $\left.\lambda\right|_{\mathscr{O}_{\mathbb{G}}(\Phi \backslash \mathbb{G} / \Phi)}=f$.

- $\lambda$ is a $\Phi$-invariant functional on $\mathscr{O}_{\mathbb{G}}(\Phi \backslash \mathbb{G})$ in the sense of Definition 3.3.

Proof. Let us first prove that there exists at least one $\Phi$-invariant extension of $f$. By virtue of Theorem 5.3, we see $\mu:=f \circ \mathbb{E}_{\ell}^{\Phi} \circ \mathbb{E}_{r}^{\Phi}$ is a $\Phi$-bi-invariant functional on $\mathscr{O}(\mathbb{G})$. Thus by Theorem 3.4 we see that $\lambda:=\left.\mu\right|_{\mathscr{O}_{\mathbb{G}}(\Phi \backslash \mathbb{G})}$ is a $\Phi$-invariant functional on $\mathscr{O}_{\mathbb{G}}(\Phi \backslash \mathbb{G})$. Clearly $\left.\lambda\right|_{\mathscr{O}_{\mathbb{G}}(\Phi \backslash \mathbb{G} / \Phi)}=f$, which proves that there exists at least one $\Phi$-invariant extension of $f$.

Suppose $\lambda^{\prime}: \mathscr{O}_{\mathbb{G}}(\Phi \backslash \mathbb{G}) \longrightarrow \mathbb{C}$ be another $\Phi$-invariant extension of $f$. Let us suppose that $\mu_{1}: \mathscr{O}(\mathbb{G}) \longrightarrow \mathbb{C}$ be the unique $\Phi$-bi-invariant extension of $f$ given by Theorem 5.3 , and $\mu_{2}: \mathscr{O}(\mathbb{G}) \longrightarrow \mathbb{C}$ be the unique $\Phi$-bi-invariant extension of $\lambda^{\prime}$ as given by Theorem 3.4. Since $\left.\mu_{2}\right|_{\mathscr{O}_{\mathbb{G}}(\Phi \backslash \mathbb{G} / \Phi)}=\left.\lambda^{\prime}\right|_{\mathscr{O}_{\mathbb{G}}(\Phi \backslash \mathbb{G} / \Phi)}=f$, this implies that $\mu_{2}$ is also a $\Phi$-bi-invariant extension of $f$. By the uniqueness of such an extension as shown in Theorem 5.3, we must have $\mu_{1}=\mu_{2}$ which in turn implies that $\lambda^{\prime}=\lambda$.

Theorem 5.3 and Corollary 5.4 together yield:

All functionals on the ${ }^{*}$-algebra of $\Phi$-bi-invariant functions on $\mathbb{G}$ are precisely the restrictions of $\Phi$-bi-invariant functionals on $\mathscr{O}(\mathbb{G})$. Hence they are also restrictions of $\Phi$-invariant functionals on the corresponding right coidalgebra.

5.2. Convolution of functionals on the *-algebra of $\Phi$-bi-invariant functions on $\mathbb{G}$. In this subsection, we again consider the ${ }^{*}$-algebra of $\Phi$-bi-invariant functions on $\mathbb{G}$ denoted by $\mathscr{O}_{\mathbb{G}}(\Phi \backslash \mathbb{G} / \Phi)$, as defined in Definition 5.1. We will define a coproduct on $\mathscr{O}_{\mathbb{G}}(\Phi \backslash \mathbb{G} / \Phi)$, which will turn it into a *-bi-algebra.

Definition 5.5. Define $\widetilde{\Delta}: \mathscr{O}(\mathbb{G}) \longrightarrow \mathscr{O}(\mathbb{G}) \otimes \mathscr{O}(\mathbb{G})$ by

$$
\widetilde{\Delta}(x):=(\mathrm{id} \otimes \Phi \otimes \mathrm{id})\left(\Delta^{(2)}(x)\right), \quad x \in \mathscr{O}_{\mathbb{G}}(\Phi \backslash \mathbb{G} / \Phi),
$$

where $\Delta^{(2)}=(\mathrm{id} \otimes \Delta) \circ \Delta$.

Lemma 5.6. The triple $\left(\mathscr{O}_{\mathbb{G}}(\Phi \backslash \mathbb{G} / \Phi),\left.\widetilde{\Delta}\right|_{\mathscr{O}_{\mathbb{G}}(\Phi \backslash \mathbb{G} / \Phi)},\left.\varepsilon\right|_{\mathscr{O}_{\mathbb{G}}(\Phi \backslash \mathbb{G} / \Phi)}\right)$ is a hyper-bialgebra (in the sense of [FS00]), i.e., 
(1) $\mathscr{O}_{\mathbb{G}}(\Phi \backslash \mathbb{G} / \Phi)$ is a unital *-algebra;

(2) the triple $\left(\mathscr{O}_{\mathbb{G}}(\Phi \backslash \mathbb{G} / \Phi),\left.\widetilde{\Delta}\right|_{\mathscr{O}_{\mathbb{G}}(\Phi \backslash \mathbb{G} / \Phi)},\left.\varepsilon\right|_{\mathscr{O}_{\mathbb{G}}(\Phi \backslash \mathbb{G} / \Phi)}\right)$ is a coalgebra;

(3) the comultiplication $\left.\widetilde{\Delta}\right|_{\mathscr{O}_{\mathbb{G}}(\Phi \backslash \mathbb{G} / \Phi)}$ is completely positive and the counit $\varepsilon_{\mathscr{O}_{\mathbb{G}}(\Phi \backslash \mathbb{G} / \Phi)}$ is $a^{*}$-algebra homomorphism.

Proof. It follows easily that $\widetilde{\Delta}$ is completely positive and coassociative. We only need to show that $\widetilde{\Delta}\left(\mathscr{O}_{\mathbb{G}}(\Phi \backslash \mathbb{G} / \Phi)\right) \subset \mathscr{O}_{\mathbb{G}}(\Phi \backslash \mathbb{G} / \Phi) \otimes \mathscr{O}_{\mathbb{G}}(\Phi \backslash \mathbb{G} / \Phi)$. So let $x \in$ $\mathscr{O}_{\mathbb{G}}(\Phi \backslash \mathbb{G} / \Phi)$. We have

$$
\begin{aligned}
\widetilde{\Delta}(x) & =\left(\mathbb{E}_{r}^{\Phi} \otimes \mathrm{id}\right)\left(\Delta^{(2)}(x)\right) \\
& =\left(\mathbb{E}_{r}^{\Phi} \otimes \mathrm{id}\right)\left(\Delta\left(\mathbb{E}_{r}^{\Phi} \circ \mathbb{E}_{\ell}^{\Phi}(x)\right)\right) \\
& =\left(\mathbb{E}_{r}^{\Phi} \circ \mathbb{E}_{\ell}^{\Phi} \otimes \mathbb{E}_{r}^{\Phi}\right)(\Delta(x)) \quad(\text { by }(\text { a }) \text { of Lemma 3.1) } \\
& =\left(\mathbb{E}_{\ell}^{\Phi} \circ \mathbb{E}_{r}^{\Phi} \circ \mathbb{E}_{r}^{\Phi} \otimes \mathbb{E}_{r}^{\Phi}\right)(\Delta(x)) \quad\left(\text { using } \mathbb{E}_{r}^{\Phi} \mathbb{E}_{\ell}^{\Phi}=\mathbb{E}_{\ell}^{\Phi} \mathbb{E}_{r}^{\Phi} \text { and }\left(\mathbb{E}_{r}^{\Phi}\right)^{2}=\mathbb{E}_{r}^{\Phi}\right) \\
& =\left(\mathbb{E}_{\ell}^{\Phi} \circ \mathbb{E}_{r}^{\Phi} \otimes \mathbb{E}_{r}^{\Phi} \circ \mathbb{E}_{\ell}^{\Phi}\right)(\Delta(x)) \quad(\text { by (b) of Lemma 3.1). }
\end{aligned}
$$

From the last expression one can conclude that $\widetilde{\Delta}(x) \in \mathscr{O}_{\mathbb{G}}(\Phi \backslash \mathbb{G} / \Phi) \otimes \mathscr{O}_{\mathbb{G}}(\Phi \backslash \mathbb{G} / \Phi)$.

As a consequence we can define convolution of functionals on $\mathscr{O}_{\mathbb{G}}(\Phi \backslash \mathbb{G} / \Phi)$.

Definition 5.7. Let $f, g$ be two functionals on $\mathscr{O}_{\mathbb{G}}(\Phi \backslash \mathbb{G} / \Phi)$. We define the convolution of $f$ and $g$ as the following functional:

$$
f \star_{b i} g:=(f \otimes g) \circ \widetilde{\Delta} .
$$

Alternatively, we have $f \star_{b i} g:=f \star \Phi \star g$.

Theorem 5.8. The following holds:

(a) Let $f, g$ be functionals on $\mathscr{O}_{\mathbb{G}}(\Phi \backslash \mathbb{G} / \Phi)$ and $\lambda_{1}, \lambda_{2}$ be their unique $\Phi$-invariant extensions as functionals on $\mathscr{O}_{\mathbb{G}}(\Phi \backslash \mathbb{G}$ ) (given by Corollary 5.4). Then

$$
\left.\lambda_{1} \star_{\ell} \lambda_{2}\right|_{\mathscr{O}_{\mathbb{G}}(\Phi \backslash \mathbb{G} / \Phi)}=f \star_{b i} g .
$$

(b) Let $T: \mathscr{O}_{\mathbb{G}}(\Phi \backslash \mathbb{G} / \Phi) \longrightarrow \mathscr{O}_{\mathbb{G}}(\Phi \backslash \mathbb{G} / \Phi)$ be a linear map such that $(T \otimes$ id) $\circ \widetilde{\Delta}=\widetilde{\Delta} \circ T$. Then there exists a $\mathbb{G}$-invariant map $S: \mathscr{O}_{\mathbb{G}}(\Phi \backslash \mathbb{G}) \longrightarrow$ $\mathscr{O}_{\mathbb{G}}(\Phi \backslash \mathbb{G})$ such that $\left.S\right|_{\mathscr{O}_{\mathbb{G}}(\Phi \backslash \mathbb{G} / \Phi)}=T$.

Proof. Observe that for $x \in \mathscr{O}_{\mathbb{G}}(\Phi \backslash \mathbb{G} / \Phi)$,

$$
\begin{aligned}
\left(\lambda_{1} \star_{\ell} \lambda_{2}\right)(x) & =\left(\lambda_{1} \otimes \lambda_{2} \circ \mathbb{E}_{\ell}^{\Phi}\right)(\alpha(x)) \\
& =\left(\lambda_{1} \otimes \lambda_{2}\right)\left(\operatorname{id} \otimes \mathbb{E}_{\ell}^{\Phi}\right)(\Delta(x)) \\
& =\left(\lambda_{1} \otimes \lambda_{2}\right)\left(\mathbb{E}_{r}^{\Phi} \otimes \mathrm{id}\right)(\Delta(x)) \quad(\text { by }(\mathrm{b}) \text { of Lemma 3.1) } \\
& =\left(\lambda_{1} \otimes \lambda_{2}\right)(\widetilde{\Delta}(x)) \\
& =(f \otimes g)(\widetilde{\Delta}(x))=\left(f \star_{b i} g\right)(x),
\end{aligned}
$$


which proves (a).

To prove $(\mathrm{b})$, observe that the identity $(T \otimes \mathrm{id}) \circ \widetilde{\Delta}=\widetilde{\Delta} \circ T$ implies that the functional $f:=\varepsilon \circ T$ satisfies $(f \otimes \mathrm{id}) \circ \widetilde{\Delta}=T$. Sine $f$ is a functional on $\mathscr{O}_{\mathbb{G}}(\Phi \backslash \mathbb{G} / \Phi)$, by virtue of Corollary [5.4, it extends to a $\Phi$-invariant functional $\lambda$ on $\mathscr{O}_{\mathbb{G}}(\Phi \backslash \mathbb{G})$. Let $S:=\left(\lambda \otimes \mathbb{E}_{\ell}^{\Phi}\right) \circ \alpha$, which is a $\mathbb{G}$-invariant operator on $\mathscr{O}_{\mathbb{G}}(\Phi \backslash \mathbb{G})$, by virtue of Theorem 3.20 . Now an easy computation yields that $\left.S\right|_{\mathscr{O}_{\mathbb{G}}(\Phi \backslash \mathbb{G} / \Phi)}=T$.

\section{Summary of the ONE-TO-ONE CORRESPONDENCES}

We have established the following one-to-one correspondences.

Theorem 6.1. Let $\mathbb{G}$ be a compact quantum group, $\Phi \in \mathcal{I}$ an idempotent state on $\mathbb{G}$, and denote by $\mathbb{X}=\Phi \backslash \mathbb{G}$ the associated quantum space. Let $\delta=\left.\varepsilon\right|_{\mathscr{O}_{\mathbb{G}}(\Phi \backslash \mathbb{G})}$.

Then we have one-to-one correspondences between the following objects.

(1) semigroups of $\mathbb{G}$-invariant operators on $\mathscr{O}_{\mathbb{G}}(\Phi \backslash \mathbb{G})$ such that $\left(\delta \circ T_{t}\right)_{t \geq 0}$ is weakly continuous;

(2) $\mathbb{G}$-invariant convolution semigroups of linear functionals on $\mathscr{O}_{\mathbb{G}}(\Phi \backslash \mathbb{G})$;

(3) $\Phi$-bi-invariant convolution semigroups of linear functionals on $\mathscr{O}(\mathbb{G})$;

(4) convolution semigroups of linear functionals on $\mathscr{O}_{\mathbb{G}}(\Phi \backslash \mathbb{G} / \Phi)$.

If we add positivity, we can formulate the following one-to-one correspondences.

Theorem 6.2. Let $\mathbb{G}$ be a compact quantum group, $\Phi \in \mathcal{I}$ an idempotent state on $\mathbb{G}$. We have one-to-one correspondences between the following objects.

(1) $\mathbb{G}$-invariant quantum Markov semigroups on $\mathscr{O}_{\mathbb{G}}(\Phi \backslash \mathbb{G})$;

(2) $\mathbb{G}$-invariant convolution semigroups of states on $\mathscr{O}_{\mathbb{G}}(\Phi \backslash \mathbb{G})$;

(3) $\Phi$-bi-invariant convolution semigroups of states on $\mathscr{O}(\mathbb{G})$;

(4) convolution semigroups of states on $\mathscr{O}_{\mathbb{G}}(\Phi \backslash \mathbb{G} / \Phi)$.

All these semigroups are furthermore characterized by their derivatives at $t=0$.

Definition 6.3. Let $\mathcal{A}$ be a unital *-algebra and $\phi: \mathcal{A} \rightarrow \mathbb{C}$ a state. A linear functional $\psi: \mathcal{A} \rightarrow \mathbb{C}$ is called a $\phi$-generating functional, if

(1) $\psi$ is normalised, i.e., $\psi(1)=0$;

(2) $\psi$ is hermitian, i.e., $\psi\left(a^{*}\right)=\overline{\psi(a)}$, for all $a \in \mathcal{A}$;

(3) $\psi$ is $\phi$-conditionally positive, i.e., $\psi\left(a^{*} a\right) \geq 0$ for all $a \in \mathcal{A}$ with $\phi\left(a^{*} a\right)=$ 0 .

Theorem 6.4. Under the same hypotheses as Theorem 6.2, the objects in Theorem 6.2 are furthermore in one-to-one correspondence with

(1) $\mathbb{G}$-invariant quantum Markov semigroups on $\mathscr{O}_{\mathbb{G}}(\Phi \backslash \mathbb{G})$;

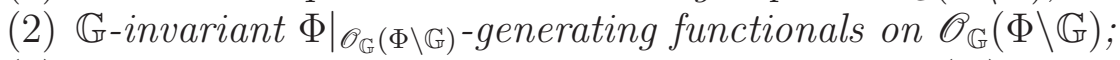

(3) $\Phi$-bi-invariant on $\Phi$-generating functionals on $\mathscr{O}(\mathbb{G})$;

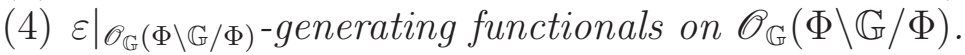


In the examples in the next section we will determine the $\left.\varepsilon\right|_{\mathscr{O}_{\mathbb{G}}}(\Phi \backslash \mathbb{G} / \Phi)$-generating functionals on $\mathscr{O}_{\mathbb{G}}(\Phi \backslash \mathbb{G} / \Phi)$ for the case where $\mathbb{G}$ is one of the orthogonal quantum groups $O_{N}, O_{N}^{*}$ or $O_{N}^{+}$and $\Phi$ is the idempotent state such that $\mathbb{X}=\Phi \backslash \mathbb{G}$ is one of the quantum spheres $S^{N-1}, S_{*}^{N-1}$, or $S_{+}^{N-1}$.

\section{MARKOV SEMIGROUPS ON QUANTUM SPHERES}

We know that orthogonal group $O_{N}$ is the isometry group of sphere $S^{N-1}$. There exist quantum versions, or "liberated" versions, of the orthogonal group and the sphere. These are given by their universal $\mathrm{C}^{*}$-algebras which are defined as follows Ba16]:

$$
\begin{gathered}
C^{u}\left(S_{+}^{N-1}\right)=C^{*}\left(x_{1}, \cdots, x_{N} \mid x_{i}=x_{i}^{*}, \sum_{i} x_{i}^{2}=1\right) \\
C^{u}\left(O_{N}^{+}\right)=C^{*}\left(\left(u_{i j}\right)_{i, j=1, \ldots, N} \mid u=\bar{u}, u^{t}=u^{-1}\right) \\
C^{u}\left(S_{*}^{N-1}\right)=C^{u}\left(S_{+}^{N-1}\right) /<a b c=c b a, \forall a, b, c \in x_{i}> \\
C^{u}\left(O_{N}^{*}\right)=C^{n}\left(O_{N}^{+}\right) /<a b c=c b a, \forall a, b, c \in u_{i j}>.
\end{gathered}
$$

We will use the notation $S_{\times}^{N-1}$ denote the three spheres above associated to the three quantum isometry groups $O_{N}^{\times}, \times \in\{\emptyset, *,+\}$ (where $\emptyset$ stands for no symbol). There exist unique actions $\alpha_{\times}^{u}: C^{u}\left(S_{\times}^{N-1}\right) \rightarrow C^{u}\left(S_{\times}^{N-1}\right) \otimes C^{u}\left(O_{N}^{\times}\right)$ of the three families orthogonal quantum groups on the corresponding spheres, such that $\alpha^{u}\left(x_{i}\right)=\sum_{j=1}^{N} x_{j} \otimes u_{j i}$ for $i \in\{1, \ldots, N\}$. Every such "universal" action comes with a "reduced" action $\alpha_{\times}^{r}: C\left(S_{\times}^{N-1}\right) \rightarrow C\left(S_{\times}^{N-1}\right) \otimes C\left(O_{N}^{\times}\right)$and a Hopf-*-algebraic action $\alpha_{\times}^{H}: \mathscr{O}_{\mathbb{G}}\left(S_{\times}^{N-1}\right) \rightarrow \mathscr{O}_{\mathbb{G}}\left(S_{\times}^{N-1}\right) \otimes \mathscr{O}\left(O_{N}^{\times}\right)$, cf. [DC16].

Banica [Ba16, Proposition 5.8] showed that the reduced function algebras of these spheres can regarded as subalgebras of the reduced function algebras of orthogonal groups. I.e. if we identify $x_{i}=u_{1 i}$, then $C\left(S_{\times}^{N-1}\right) \subset C\left(O_{N}^{\times}\right)$. One can check that $C\left(S_{\times}^{N-1}\right)$ is a coidalgebra of $C\left(O_{N}^{\times}\right)$, so we can define the corresponding idempotent state $\Phi$ such that the associated left, right, and two-sided conditional expectations $\mathbb{E}_{\ell}^{\Phi}, \mathbb{E}_{r}^{\Phi}, \mathbb{E}_{b i}^{\Phi}$ satisfy:

$$
\begin{gathered}
\mathscr{O}\left(S_{\times}^{N-1}\right)=\mathscr{O}_{O_{N}^{\times}}\left(\Phi \backslash O_{N}^{\times}\right)=\mathbb{E}_{\ell}^{\Phi}\left(\mathscr{O}\left(O_{N}^{\times}\right)\right)=*_{-} \operatorname{alg}\left\{u_{11}, \ldots, u_{1 N}\right\}, \\
S\left(\mathscr{O}\left(S_{\times}^{N-1}\right)\right)=\mathscr{O}_{O_{N}^{\times}}\left(O_{N}^{\times} / \Phi\right)=\mathbb{E}_{r}^{\Phi}\left(\mathscr{O}\left(O_{N}^{\times}\right)\right)={ }^{*}-\operatorname{alg}\left\{u_{11}, \ldots, u_{N 1}\right\}, \\
\mathscr{O}\left(S_{\times}^{N-1}\right) \cap S\left(\mathscr{O}\left(S_{\times}^{N-1}\right)\right)=\mathscr{O}_{O_{N}^{\times}}\left(\Phi \backslash O_{N}^{\times} / \Phi\right)=\mathbb{E}_{b i}^{\Phi}\left(\mathscr{O}\left(O_{N}^{\times}\right)\right)=*_{-} \operatorname{alg}\left\{u_{11}\right),
\end{gathered}
$$

(where $S$ denotes the antipode of $\mathscr{O}\left(O_{N}^{\times}\right)$).

We know that in the classical case $S^{N-1} \cong O_{N-1} \backslash O_{N}$. Banica, Skalski, and Soltan [BSS12] have shown that $S_{+}^{N-1}$ is not equal to the quotient $O_{N-1}^{+} \backslash O_{N}^{+}$. We will now show that the half-liberated and the free spheres can not be obtained as quotient spaces. 
Proposition 7.1. There exists no quantum subgroup $\mathbb{K}$ of $O_{N}^{+}$(or $O_{N}^{*}$, resp.) such that $S_{+}^{N-1} \cong O_{N}^{+} / \mathbb{K}$ (or $S_{*}^{N-1} \cong O_{N}^{*} / \mathbb{K}$, resp.) as left coidalgebras.

Proof. We start with the free sphere.

If such a quantum subgroup existed, then it would be of Kac type, and therefore its Haar idempotent $\Phi_{\mathbb{K}}=h_{\mathbb{K}} \circ \theta$ would be tracial. We will now show that the idempotent state associated to $\mathscr{O}\left(S_{+}^{N-1}\right)$ by Theorem 1.3 is not a trace.

Let $\mathbb{E}_{b i}^{\Phi}$ denote the conditional expectation in $\mathscr{O}\left(O_{N}^{+}\right)$onto the ${ }^{*}$-subalgebra of $\mathscr{O}\left(O_{N}^{+}\right)$generated by $u_{11}$, then we have $\Phi=\varepsilon \circ \mathbb{E}_{l}^{\Phi}=\varepsilon \circ \mathbb{E}_{b i}^{\Phi}$.

$\mathbb{E}_{b i}^{\Phi}$ is the orthogonal projection onto ${ }^{*}$-subalgebra genrated by $u_{11}$ for the inner product $\langle a, b\rangle=h\left(a^{*} b\right)$, and since we can compute the values of the Haar state on products of the algebraic generators $u_{11}, \ldots, u_{N N}$ using the Weingarten calculus, we can compute $\mathbb{E}_{b i}^{\Phi}$ and then $\Phi$. We find

$$
\mathbb{E}_{b i}^{\Phi}\left(u_{22} u_{11} u_{22}\right)=0
$$

since $h_{O_{N}^{+}}\left(u_{11}^{k} u_{22} u_{11} u_{22}\right)=0$ for all $k \in \mathbb{N}$ (there are no matching non-crossing pairings) and

$$
\mathbb{E}_{b i}^{\Phi}\left(u_{11} u_{22}^{2}\right)=u_{11} \mathbb{E}_{b i}^{\Phi}\left(u_{22}^{2}\right)=\frac{(N-2) u_{11}+u_{11}^{3}}{(N-1)^{2}}
$$

since

$$
h_{O_{N}^{+}}\left(u_{22}^{2}\right)=\frac{1}{N} \quad \text { and } \quad h_{O_{N}^{+}}\left(u_{11}^{2} u_{22}^{2}\right)=\frac{1}{N^{2}-1}
$$

If follows that

$$
\Phi\left(u_{11} u_{22}^{2}\right)=\varepsilon\left(\frac{(N-2) u_{11}+u_{11}^{3}}{(N-1)^{2}}\right)=\frac{1}{N-1} \neq 0=\Phi\left(u_{22} u_{11} u_{22}\right) .
$$

The case of the half-liberated sphere $S_{*}^{N-1}$ is similar. Let us recall that a pairing is called "balanced," if each pair connects a black leg to a while leg, when we label its legs alternately black and white: $\bullet \circ \bullet \cdots .$. Denote the set of balanced pairings of $n$ elements by $P_{2}^{*}(n)$. The Weingarten formula for $O_{N}^{*}$ uses balanced pairings. The balanced pairings $P_{2}^{*}(4)$ and the non-crossing pairings $\mathrm{NC}_{2}(4)$ of four elements are the same. Thus, we get again the same values for Haar state in the half-liberated case,

$$
\begin{gathered}
h_{O_{N}^{*}}\left(u_{11}^{k} u_{22} u_{11} u_{22}\right)=0, \quad k=0,1, \ldots, \\
h_{O_{N}^{*}}\left(u_{22}^{2}\right)=\frac{1}{N} \quad \text { and } \quad h_{O_{N}^{*}}\left(u_{11}^{2} u_{22}^{2}\right)=\frac{1}{N^{2}-1},
\end{gathered}
$$

and we get the same conclusion.

We want to compute the eigenvalues and eigenspaces of $O_{N}^{\times}$-invariant Markov semigroups on $\mathscr{O}\left(S_{\times}^{N-1}\right)$. First, we will give a decomposition of the Hilbert spaces 
$L^{2}\left(\left(\mathscr{O}\left(S_{\times}^{N-1}\right), h\right)\right.$, and $L^{2}\left(\left(\mathscr{O}\left(O_{N-1}^{\times}\right), h\right)\right.$ where $h$ denotes the Haar state on $C\left(O_{N}^{\times}\right)$, restricted to $C\left(S_{\times}^{N-1}\right)$. Set

$$
\begin{gathered}
E_{k}=\operatorname{span}\left\{u_{i_{1} j_{1}} \cdots u_{i_{r} j_{r}}: r \leq k\right\} \subset L_{2}\left(\mathscr{O}\left(O_{N}^{\times}\right), h\right) ; \\
H_{k}=\operatorname{span}\left\{x_{i_{1}} \cdots x_{i_{r}}: r \leq k\right\} \subset L_{2}\left(\mathscr{O}\left(S_{\times}^{N-1}\right), h\right) ; \\
V_{k}=E_{k} \cap E_{k-1}^{\perp} ; \quad D_{k}=H_{k} \cap H_{k-1}^{\perp} ; \quad d_{k}=\operatorname{dim} D_{k} .
\end{gathered}
$$

Then $L_{2}\left(\mathscr{O}\left(S_{\times}^{N-1}\right), h\right)=\bigoplus_{k \in \mathbb{N}} D_{k}$ and $L_{2}\left(\mathscr{O}\left(O_{N}^{\times}\right), h\right)=\bigoplus_{k \in \mathbb{N}} V_{k}$. Furthermore $H_{k}=\mathbb{E}_{\ell}^{\Phi}\left(E_{k}\right)$, and thus $D_{k}=\mathbb{E}_{\ell}^{\Phi}\left(V_{k}\right)$.

Take a complete set $\left\{u^{\pi}: \pi \in \operatorname{Irr}\left(O_{N}^{\times}\right)\right\}$of mutually inequivalent, irreducible unitary representations. We know that the matrix $u=\left(u_{i j}\right)$ is an irreducible unitary representation of $O_{N}^{\times}$whose coefficients generate the function algebra. We can decompose its tensor powers $u^{\bigotimes s}=\bigoplus_{\pi \in I_{s}} n_{\pi}^{s} u^{\pi}$, where $n_{\pi}^{s}$ denotes the multiplicity of $u^{\pi}$, and we used the notation $I_{s}:=\left\{\pi \in \operatorname{Irr}\left(O_{N}^{\times}\right): n_{\pi}^{s} \geq 1\right\}$.

Then, for any $s \geq 2$, we define

$$
u^{(s)}:=\bigoplus_{\pi \in J_{s}} u^{\pi}, \quad \text { where } J_{s}=I_{s} \backslash \cup_{0 \leq i \leq s-1} I_{i} .
$$

In other words, $u^{(s)}$ is the direct sum of the "new" irreducible corepresentations in the decomposition of $u^{\bigotimes s}$, those which did not appear in the decompositions of $u^{\bigotimes i}, \forall i<s$.

Since the linear space spanned by coefficients of $\left\{u^{\bigotimes i}\right\}_{0 \leq i \leq s}$ is $E_{s}$, by decomposition $E_{s}=\operatorname{span}\left\{u_{p q}^{\pi}: \pi \in I_{i}, 0 \leq i \leq s\right\}$. Thus by definition, the linear space spanned by coefficients of $u^{(s)}$ is $V_{s}$.

For the free case, by the fusion rule of $O_{N}^{+}$, we know that $I_{s}=\{s, s-2, s-4, \ldots\}$, therefore $J_{s}=\{s\}$. So $u^{(s)}$ is exactly the $s^{\text {th }}$ irreducible unitary corepresentation of $O_{N}^{+}$. But for other two cases, $u^{(s)}$ defined here may not be irreducible, but it is the direct sum of some mutually inequivalent irreducible unitary representations.

We state the argument above as a proposition:

Proposition 7.2. There exists a sequence of unitary corepresentations $\left(u^{(s)}\right)_{s \in \mathbb{N}}$ of $O_{N}^{\times}$, such that the non-zero coefficients of $u^{(s)}$ are linearly independent and span $V_{s}$. In the free case, $u^{(s)}$ is irreducible.

The following lemma is the main step for characterising the idempotent state $\Phi$.

Lemma 7.3. There exists a basis for the Hilbert space $D_{s}$ associated to the corepresentation $u^{(s)}$, such that we get

$$
\Phi(s):=\left(\Phi\left(u_{j k}^{(s)}\right)\right)_{1 \leq j, k \leq d_{s}}=\delta_{j 1} \delta_{i 1} .
$$

if we write $u^{(s)}=\left(u_{j k}^{(s)}\right)_{1 \leq j, k \leq d_{s}}$ w.r.t. to this basis. In other words, the corepresentation $u^{(s)}$ is unitarily equivalent to one for which applying $\Phi$ to it coefficient-wise produces a matrix with entry 1 in the upper left corner and 0 everywhere else. 
Proof. Since $\Phi$ is idempotent state, we can easily check that

$$
\left\|\Phi_{s}\right\| \leq 1 \text { and } \Phi_{s}^{2}=\Phi_{s},
$$

which means that $\Phi_{s}$ is a projection in $B\left(D_{s}\right)$. We know that every projection matrix can be written as a diagonal matrix with coefficients 1 and 0 by choosing some suitable basis. So

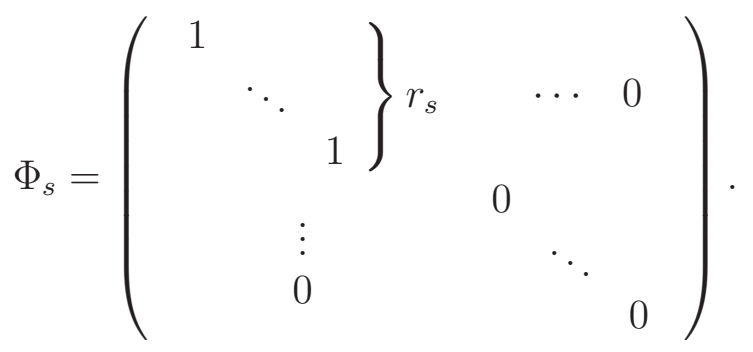

Denote the rank of this matrix by $r_{s}$. For all $k$, we take the basis of $D_{k}$ as above, so that for $0 \leq i \leq r_{k}, \Phi\left(u_{i i}^{(s)}\right)=1$; otherwise $\Phi\left(u_{i j}^{(s)}\right)=0$. Then for any $s \in \mathbb{N}$,

$$
\mathbb{E}_{b i}^{\Phi}\left(u_{i j}^{(s)}\right)=\sum_{p, q} \Phi\left(u_{i p}^{(s)}\right) u_{p q}^{(s)} \Phi\left(u_{q j}^{(s)}\right)= \begin{cases}u_{i j}^{(s)} & \text { if } 1 \leq i, j \leq r_{s} \\ 0 & \text { otherwise. }\end{cases}
$$

Moreover, the conditional expectation $\mathbb{E}_{b i}^{\Phi}$ sends $E_{s}$ onto

$$
\operatorname{Pol}_{s}\left(u_{11}\right):=\left\{1, u_{11}, u_{11}^{2}, \cdots, u_{11}^{s}\right\} .
$$

Thus,

$$
\operatorname{dim}\left(\operatorname{Pol}_{s}\left(u_{11}\right)\right)=\operatorname{dim}\left(\mathbb{E}_{b i}^{\Phi}\left(E_{s}\right)\right)=\operatorname{dim}\left(\mathbb{E}_{b i}^{\Phi}\left(\bigoplus_{k=0}^{s}\left(V_{k}\right)\right)\right)=\sum_{k=0}^{s} r_{k}^{2},
$$

which implies

$$
r_{s}^{2}=\operatorname{dim}\left(\operatorname{Pol}_{s}\left(u_{11}\right)\right)-\operatorname{dim}\left(\operatorname{Pol}_{s-1}\left(u_{11}\right)\right)=s+1-s=1 .
$$

This theorem tells us that $u_{11}^{(s)}=\mathbb{E}_{b i}^{\Phi}\left(u_{11}^{(s)}\right) \in \operatorname{Pol}_{s}\left(u_{11}\right)$. Moreover, the algebra *-alg$\left\{u_{11}\right\}$ as a subalgebra of $\mathscr{O}\left(O_{N}^{\times}\right)$and can be identified with the algebra of polynomials on the interval $[-1,1]$. Therefore, there exists $q_{k}^{\times} \in \operatorname{Pol}[-1,1]$ such that $q_{k}^{\times}\left(u_{11}\right)=u_{11}^{(k)}$. Since $h_{O_{N}^{\times}}\left(q_{n}^{\times}\left(u_{11}\right) q_{m}^{\times}\left(u_{11}\right)\right)=h_{O_{N}^{\times}}\left(u_{11}^{(n)} u_{11}^{(m)}\right)=C \delta_{n m}$, $\left(q_{s}\right)_{s \in \mathbb{N}}$ is a family of orthogonal polynomials. The measure of orthogonality of these polynomials is the probability meeasure obtained by evaluating the spectral measure of $u_{11}$ in the Haar state. Since $u_{11}$ is hermitian and we have $\left\|u_{11}\right\| \leq 1$, we get a measure that is supported on $[-1,1]$ (which explains why we consider only the values of our polynomials on this interval).

The restriction of the counit to ${ }^{*}-\operatorname{alg}\left\{u_{11}\right\}$ corresponds to evaluation of a polynomial in the boundary point 1 , i.e. $\varepsilon\left(p\left(u_{11}\right)\right)=p(1), \forall p \in \operatorname{Pol}[-1,1]$. Therefore we obtain the following result, in the same manner as in CFK14, Proposition $10.1]$. 
Proposition 7.4. CFK14, Proposition 10.1]. Let $\psi$ be a conditionally positive functional on $\operatorname{Pol}\left[u_{11}\right]$. Then there exist a unique pair $(b, \nu)$ consisting of a real number $b \geq 0$ and a finite measure $\nu$ on $[-1,1]$ such that

$$
\psi(p)=-b p^{\prime}(1)+\int_{-1}^{1} \frac{p(x)-p(1)}{x-1} \mathrm{~d} \nu(x)
$$

for any polynomial $p$. Conversely, every $\psi$ of this form is conditionally positive.

Applying the above proposition, we can compute the eigenvalues of Markov semigroups.

Theorem 7.5. For any $O_{N}^{\times}$-invariant strongly continuous Markov semigroup $\left(T_{t}\right)_{t \geq 0}$ on sphere $\mathscr{O}\left(S_{\times}^{N-1}\right)$, there exists a pair $(b, \nu)$, with $b$ a positive number and $\nu$ a finite measure on $[-1,1]$, such that the generator $L$ of $\left(T_{t}\right)_{t \geq 0}$ satisfying,

$$
L\left(x_{s}\right)=\lambda_{s} x_{s} \quad \forall x_{s} \in D_{s},
$$

where

$$
\lambda_{s}=-b\left(q_{s}^{\times}\right)^{\prime}(1)+\int_{-1}^{1} \frac{q_{s}^{\times}(x)-1}{x-1} \mathrm{~d} \nu(x) .
$$

Moreover, if $T_{0}=\mathrm{id}$, then for any $t \geq 0, T_{t}\left(x_{s}\right)=e^{t \lambda_{s}} x_{s}, \forall x_{s} \in D_{s}$.

Proof. Theorem 4.6 guarantees the existence of generator operator $L$, and the Markov property makes $\psi:=\varepsilon \circ L$ conditionally positive.

By Lemma 7.3, we can compute $\mathbb{E}_{\ell}^{\Phi}\left(u_{i j}^{(k)}\right)=\delta_{1 i} u_{1 j}^{(k)}$, which implies $D_{s}=$ $\mathbb{E}_{\ell}^{\Phi}\left(V_{k}\right)=\operatorname{span}\left\{u_{1 j}^{(s)}: 1 \leq j \leq d_{k}\right\}$. Then for any $u_{1 j}^{(s)} \in D_{s}$,

$$
L\left(u_{1 j}^{(s)}\right)=\sum_{k} \psi\left(u_{1 k}^{(s)}\right) \mathbb{E}_{\ell}^{\Phi}\left(u_{k j}^{(s)}\right)=\psi\left(u_{11}^{(s)}\right) u_{1 j}^{(s)}
$$

Now, we just need to consider $\left.\psi\right|_{*_{-}} \lg _{\left(u_{11}\right)}$ which induces the pair $(b, \nu)$ by Proposition 7.4. By linearity of $L$, we can get the eigenvalues for $D_{s}$,

$$
\lambda_{s}=\psi\left(u_{11}^{(s)}\right)=-b\left(q_{s}^{\times}\right)^{\prime}(1)+\int_{-1}^{1} \frac{q_{s}^{\times}(x)-1}{x-1} \mathrm{~d} \nu(x),
$$

since $q_{s}^{\times}(1)=\varepsilon\left(u_{11}^{(s)}\right)=1$.

We point out here that we have three different families of orthogonal polynomials $\left\{q_{s}^{\times}(x)\right\}$ associated to $S_{\times}^{N-1}$, since the Haar states $h_{O_{N}^{\times}}$depend on $\times \in$ $\{\emptyset, *,+\}$. We will desccribe these orthogonal polynomials case by case.

7.1. The classical sphere $S^{N-1}$. Here, $\left(q_{s}(x)\right)_{s \in \mathbb{N}}$ means the family of the orthogonal polynomials associated to classial sphere. It is well known that the distribution of $u_{11}^{2}$ for the classical sphere is the beta distribution with parameters $(1 / 2,(N-1) / 2)$. In other words,

$$
h_{S^{N-1}}\left(\phi\left(u_{11}^{2}\right)\right)=C \int_{0}^{1} \phi(t) \frac{1}{\sqrt{t}}(1-t)^{\frac{N-3}{2}} d t,
$$


where $C=\frac{\Gamma\left(\frac{N}{2}\right)}{\Gamma\left(\frac{1}{2}\right) \Gamma\left(\frac{N-1}{2}\right)}$. The integral vanishes on the odd polynomials, i.e. $h_{S^{N-1}}\left(u_{11}^{2 k+1}\right)=$

0. Therefore $h_{S^{N-1}}\left(\frac{f\left(u_{11}\right)+f\left(-u_{11}\right)}{2}\right)=h_{S^{N-1}}\left(f\left(u_{11}\right)\right)$.

$$
\begin{aligned}
h_{S^{N-1}}\left(f\left(u_{11}\right)\right) & =h_{S^{N-1}}\left(\frac{f\left(u_{11}\right)+f\left(-u_{11}\right)}{2}\right) \\
& =C \int_{-1}^{1} f(t)(1-t)^{\frac{N-3}{2}}(1+t)^{\frac{N-3}{2}} \mathrm{~d} t
\end{aligned}
$$

The spectral measure of $u_{11}$ is the probability measure on the interval $[-1,1]$ :

$$
\mu(d t)=C(1-t)^{\frac{N-3}{2}}(1+t)^{\frac{N-3}{2}} d t,
$$

whose family of orthogonal polynomials is well known. Namely, we get the Jacobi polynomials (or ultraspherical polynomials) with parameters $\alpha=\beta=(N-3) / 2$, which we will denote by $\left(J_{s}\right)_{s \in \mathbb{N}}$.

Recall that Jacobi polynomials are given by:

$$
J_{s}(x)=\sum_{r=0}^{s}\left(\begin{array}{c}
s+(N-3) / 2 \\
r
\end{array}\right)\left(\begin{array}{c}
s+(N-3) / 2 \\
(N-3) / 2-r
\end{array}\right)\left(\frac{x-1}{2}\right)^{s-r}\left(\frac{x+1}{2}\right)^{r} .
$$

Their orthogonality relation is given by

$$
\int_{-1}^{1} J_{k}(x) J_{m}(x) \mu(d t)=\delta_{k m} \cdot C \frac{2^{N-2}}{2 k+N-2} \frac{\Gamma(k+(N-1) / 2)^{2}}{\Gamma(k+N-2) n !} .
$$

Moreover, they satisfy the differential equation

$$
\left(1-x^{2}\right) J_{s}^{\prime \prime}(x)-x(N-1) J_{s}^{\prime}(x)=-s(s+N-2) J_{s}(x) .
$$

We need these polynomials in the form $q_{s}(x)=J_{s}(x) / J_{s}(1)$.

Therefore,

$$
q_{s}^{\prime}(1)=\frac{s(s+N-2)}{N-1} .
$$

We can relate our result to the Morkov sequence problem. For a given orthonormal basis $\left\{f_{0}=\mathbf{1}, f_{1}, f_{2}, \ldots\right\}$ of the $L^{2}$-space of some probability space, this problem of ask for the classification of all sequences $\left(\lambda_{n}\right)_{n \geq 0}$ such that $K\left(f_{n}\right)=\lambda_{n} f_{n}$ defines Markov operator, cf. [BaM18]. In [Bo54, Theorem 2], Bochner answered this problem for the Jacobi polynomials. Since we found that the Jacobi polynomials are the eigevectors for any $O_{N}$-invariant Markov semigroup on $S^{N-1}$, our Theorem 7.5 recovers [Bo54, Theorem 3].

7.2. The half-liberated sphere $S_{*}^{N-1}$. Next we consider the half-liberated sphere $S_{*}^{N-1}$.

Banica [Ba16, Propsition 6.6] determined the law of $x_{i_{1}} \cdots x_{i_{k}}$ with respect to the Haar state $h_{S_{*}^{N-1}}=\left.h_{O_{N}^{*}}\right|_{C\left(S_{*}^{N-1}\right)}$ (there is a small misprint in [Ba16, Propsition 6.6], which we correct below). 
Proposition 7.6. The half-liberated integral of $x_{i_{1}} \cdots x_{i_{k}}$ vanishes, unless each index a appears the same number of times at odd and even positions in $i_{1}, \ldots, i_{k}$. We have

$$
\int_{S_{*}^{N-1}} x_{i_{1}} \cdots x_{i_{k}} d x=\frac{(N-1) ! \ell_{1} ! \cdots \ell_{n} !}{\left(N+\sum \ell_{i}-1\right) !}
$$

where $\ell_{i}$ denotes this number of common occurrences of $i$ in the $k$-tuple $\left(i_{1}, \ldots, i_{k}\right)$.

This proposition allows to describe the spectral distribution of $u_{11}=x_{1}$ w.r.t. the Haar state.

Corollary 7.7. The distribution of $u_{11}$ in the half-liberated case is given by:

$$
h_{S_{*}^{N-1}}\left(f\left(u_{11}\right)\right)=\int_{-1}^{1} f(t) \mu(d t), \quad \forall f \in C([-1,1])
$$

where $\mu(d t)=(N-1)\left(1-t^{2}\right)^{N-2}|t| d t$.

Proof. This proof repeats the arguments of [Ba16, Propsitions 6.5 and 6.6].

Let $C=\frac{2^{N}}{2 N \pi^{N}} \cdot \Gamma(N+1)=\left(\frac{2}{\pi}\right)^{N}(N-1)$ !, then

$$
\begin{aligned}
h_{S_{*}^{N-1}}\left(x^{2 k}\right)= & \int_{S_{\mathbb{C}}^{N-1}}\left|z_{1}\right|^{2 k} d z \\
= & C \int_{S_{\mathbb{R}}^{2 N-1}}\left(x_{1}^{2}+y_{1}^{2}\right)^{k} d(x, y) \\
= & C \int_{0}^{\pi / 2} \int_{0}^{\pi / 2}\left(\cos ^{2} \theta_{1}+\sin ^{2} \theta_{1} \cos ^{2} \theta_{2}\right)^{k} \sin ^{2 N-2} \theta_{1} \sin ^{2 N-3} \theta_{2} d \theta_{1} d \theta_{2} \\
& \cdot \int_{0}^{\pi / 2} \sin ^{2 N-4} \theta_{3} d \theta_{3} \cdots \int_{0}^{\pi / 2} \sin \theta_{2 N-2} d \theta_{2 N-2} \cdot \int_{0}^{\pi / 2} d \theta_{2 N-1} .
\end{aligned}
$$

First we can calculate that

$$
\begin{aligned}
C^{\prime} & =C \int_{0}^{\pi / 2} \sin ^{2 N-4} \theta_{3} d \theta_{3} \cdots \int_{0}^{\pi / 2} \sin \theta_{2 N-2} d \theta_{2 N-2} \cdot \int_{0}^{\pi / 2} d \theta_{2 N-1} \\
& =\left(\frac{2}{\pi}\right)^{N}(N-1) ! \cdot\left(\frac{\pi}{2}\right)^{N-1} \frac{(2 N-4) ! !}{(2 N-3) ! !} \frac{(2 N-5) ! !}{(2 N-4) ! !} \cdots \frac{1 ! !}{2 ! !} \\
& =\frac{4}{\pi}(N-1)
\end{aligned}
$$

where $m ! !=(m-1)(m-3) \cdots 1$. Let $t=\sqrt{\cos ^{2} \theta_{1}+\sin ^{2} \theta_{1} \cos ^{2} \theta_{2}}, u=\cos \theta_{1}$, then

$$
\begin{aligned}
h_{S_{*}^{N-1}}\left(x^{2 k}\right) & =C^{\prime} \int_{0}^{1} \int_{0}^{t} t^{2 k}\left(1-t^{2}\right)^{N-2} \frac{t}{\sqrt{t^{2}-u^{2}}} d u d t \\
& =2(N-1) \int_{0}^{1} t^{2 k} \cdot\left(1-t^{2}\right)^{N-2} t d t .
\end{aligned}
$$


Since the odd moments of $u_{11}$ vanish, we have $h_{S_{*}^{N-1}}\left(f\left(u_{11}\right)\right)=h_{S_{*}^{N-1}}\left(\frac{f\left(u_{11}\right)+f\left(-u_{11}\right)}{2}\right)$. and

$$
\begin{aligned}
h_{S_{*}^{N-1}}\left(f\left(u_{11}\right)\right) & =2(N-1) \int_{0}^{1}\left(\frac{f\left(u_{11}\right)+f\left(-u_{11}\right)}{2}\right) \cdot\left(1-t^{2}\right)^{N-2} t d t \\
& =(N-1) \int_{-1}^{1} f\left(u_{11}\right)\left(1-t^{2}\right)^{N-2}|t| d t .
\end{aligned}
$$

Now we determine the family of orthogonal polynomials associated to the probability measure $\mu$ defined in Corollary 7.7.

The standard notation for hypergeometric functions is

$$
{ }_{r} F_{s}\left(\begin{array}{l}
a_{1}, \cdots, a_{r} \\
b_{1}, \cdots, b_{s}
\end{array} ; x\right)=\sum_{n=0}^{\infty} \frac{\left(a_{1}\right)_{n} \cdots\left(a_{r}\right)_{n}}{\left(b_{1}\right)_{n} \cdots\left(b_{s}\right)_{n}} \frac{x^{n}}{n !}
$$

where the shifted factorial $(a)_{n}$ is defined by

$$
(a)_{n}= \begin{cases}a(a+1) \cdots(a+n-1), & n=1,2, \cdots \\ 1, & n=0 .\end{cases}
$$

They satisfy

$$
\frac{d}{d x} r F_{s}\left(\begin{array}{c}
a_{1}, \cdots, a_{r} \\
b_{1}, \cdots, b_{s}
\end{array} ; x\right)=\frac{\prod_{i=1}^{r} a_{i}}{\prod_{j=1}^{s} b_{i}}{ }_{i} F_{s}\left(\begin{array}{c}
a_{1}+1, \cdots, a_{r}+1 \\
b_{1}+1, \cdots, b_{s}+1
\end{array} ; x\right) .
$$

And by Gauss' theorem we have

$$
{ }_{2} F_{1}\left(\begin{array}{c}
a, b \\
c
\end{array} ; 1\right)=\frac{\Gamma(c) \Gamma(c-a-b)}{\Gamma(c-a) \Gamma(c-b)} .
$$

Definition 7.8. We define the family half-liberated spherical polynomials (or "*-polynomials") by

$$
\begin{aligned}
P_{2 k}(x) & =(-1)^{k}\left(\begin{array}{c}
N+2 k-2 \\
k
\end{array}\right){ }_{2}^{-1} F_{1}\left(\begin{array}{c}
-k, N+k-1 \\
1
\end{array} ; x^{2}\right) \\
& =\sum_{r=0}^{k}(-1)^{k+r}\left(\begin{array}{c}
k \\
r
\end{array}\right)^{2}\left(\begin{array}{c}
N+2 k-2 \\
k-r
\end{array}\right)^{-1} x^{2 r}, \\
P_{2 k+1}(x) & =x \cdot(-1)^{k}(k+1)\left(\begin{array}{c}
N+2 k-1 \\
k
\end{array}\right)^{-1}{ }_{2} F_{1}\left(\begin{array}{c}
-k, N+k \\
2
\end{array} ; x^{2}\right) \\
& =\sum_{r=0}^{k}(-1)^{k+r}\left(\begin{array}{c}
k \\
r
\end{array}\right)\left(\begin{array}{c}
k+1 \\
r+1
\end{array}\right)\left(\begin{array}{c}
N+2 k-1 \\
k-r
\end{array}\right)^{-1} x^{2 r+1} .
\end{aligned}
$$


Proposition 7.9. The family of "*-polynomials" satisfies the following threeterm recurrence relation:

$$
P_{s}(x)=x P_{s-1}(x)-\omega_{s-2} P_{s-2}(x) \quad \forall s \geq 2,
$$

where $\omega_{\ell}=\frac{[(\ell+2) / 2](N-1+[\ell / 2])}{(N+\ell)(N+\ell-1)}$. Moreover, the "*-polynomials" are the orthogonal polynomials for the probability measure $\mu(d t)=(N-1)\left(1-t^{2}\right)^{N-2}|t| d t$.

Proof. We can easily check that for any $k \geq 1$,

$$
\begin{aligned}
& x P_{2 k}(x)-\frac{k(N+k-2)}{(N+2 k-2)(N+2 k-1)} P_{2 k-1}(x)=P_{2 k+1}(x), \\
& x P_{2 k-1}(x)-\frac{k(N+k-2)}{(N+2 k-2)(N+2 k-3)} P_{2 k-2}(x)=P_{2 k}(x) .
\end{aligned}
$$

Therefore the three-term recurrence relation holds.

By the Proposition 7.6, we can calculate

$$
\begin{aligned}
\int_{-1}^{1} P_{2 k}(x) \mu(d x) & =\sum_{r=0}^{k}(-1)^{k+r}\left(\begin{array}{l}
k \\
r
\end{array}\right)^{2}\left(\begin{array}{c}
N+2 k-2 \\
k-r
\end{array}\right)^{-1} \frac{(N-1) ! r !}{(N+r-1) !} \\
& =(-1)^{k}\left(\begin{array}{c}
N+2 k-2 \\
k
\end{array}\right)^{-1} \sum_{r=0}^{k} \frac{(-1)^{r} k !(N+k+r-2) !(N-1) !}{(k-r) !(N+k-2) !(N+r-1) ! r !} \\
& =(-1)^{k}\left(\begin{array}{c}
N+2 k-2 \\
k
\end{array}\right)^{-1}{ }_{2} F_{1}\left(\begin{array}{c}
-k, N+k-1 \\
N
\end{array}\right) \\
& =(-1)^{k}\left(\begin{array}{c}
N+2 k-2 \\
k
\end{array}\right)^{-1} \frac{\Gamma(N)}{\Gamma(1-k) \Gamma(N+k)} \\
& =\delta_{0 k} ;
\end{aligned}
$$

and all of the odd moments vanish, i.e., $\int_{-1}^{1} P_{2 k+1}(x) \mu(d x)=0$.

We now prove the orthogonality by induction.

Clearly, $\forall n>0, \int_{-1}^{1} P_{n}(x) P_{0}(x) \mu(d x)=0$.

Assume that for any $0 \leq k \leq s, \int_{-1}^{1} P_{n}(x) P_{k}(x) \mu(d x)=0$ holds for all $n>k$. Then consider $s+1$, and $n>s+1$. Using the three-term recurrence relation, we get

$$
\begin{aligned}
\int_{-1}^{1} P_{n}(x) P_{s+1}(x) \mu(d x) & =\int_{-1}^{1} P_{n}(x)\left(x P_{s}(x)-\omega_{s-1} P_{s-1}(x)\right) \mu(d x) \\
& =\int_{-1}^{1} x P_{n}(x) P_{s}(x) \mu(d x)+0 \\
& =\int_{-1}^{1}\left(P_{n+1}(x)+\omega_{n-1} P_{n-1}\right) P_{s}(x) \mu(d x) \\
& =0 .
\end{aligned}
$$


Moreover,

$$
\begin{aligned}
\int_{-1}^{1} P_{s}^{2}(x) \mu(d x) & =\int_{-1}^{1} P_{s}(x)\left(x P_{s-1}-\omega_{s-2} P_{s-2}\right) \mu(d x) \\
& =\int_{-1}^{1}\left(P_{s+1}(x)+\omega_{s-1} P_{s-1}(x)\right) P_{s-1}(x) \mu(d x) \\
& =\omega_{s-1} \int_{-1}^{1} P_{s-1}^{2}(x) \mu(d x)=\omega_{0} \omega_{1} \cdots \omega_{s-1}
\end{aligned}
$$

so that

$$
\int_{-1}^{1} P_{m}(x) P_{n}(x) \mu(d x)=\omega_{0} \omega_{1} \cdots \omega_{n-1} \cdot \delta_{m n} .
$$

Remark 7.10. We change the normalisation of these polynomial to get the sequence $q_{s}^{*}(x)=\frac{P_{s}(x)}{P_{s}(1)}$ which satisfies the conditions of Theorem 7.5 .

We have

$$
\begin{aligned}
P_{2 k}(1) & =(-1)^{k}\left(\begin{array}{c}
N+2 k-2 \\
k
\end{array}\right){ }_{2}^{-1} F_{1}\left(\begin{array}{c}
-k, N+k-1 \\
1
\end{array}{ }^{-1}\right) \\
& =(-1)^{k} \frac{k !(N+k-2) !}{(N+2 k-2) !} \frac{\Gamma(1) \Gamma(2-N)}{\Gamma(k+1) \Gamma(2-N-k)} \\
& =\frac{(N+k-2) !(N+k-2) !}{(N+2 k-2) !(N-2) !} \\
P_{2 k+1}(1) & =(-1)^{k}(k+1)\left(\begin{array}{c}
N+2 k-1 \\
k
\end{array}\right)^{-1}{ }_{2} F_{1}\left(\begin{array}{c}
-k, N+k \\
2
\end{array}{ }^{2}\right) \\
& =(-1)^{k}(k+1) \frac{k !(N+k-1) !}{(N+2 k-1) !} \frac{\Gamma(2) \Gamma(2-N)}{\Gamma(k+2) \Gamma(2-N-k)} \\
& =\frac{(N+k-1) !(N+k-2) !}{(N+2 k-1) !(N-2) !}
\end{aligned}
$$

Therefore

$$
\begin{gathered}
q_{2 k}^{*}(x)=(-1)^{k}\left(\begin{array}{c}
N+k-2 \\
k
\end{array}\right)^{-1}{ }_{2} F_{1}\left(\begin{array}{c}
-k, N+k-1 \\
1
\end{array} x^{2}\right) \\
q_{2 k+1}^{*}(x)=x \cdot(-1)^{k}(k+1)\left(\begin{array}{c}
N+k-2 \\
k
\end{array}\right){ }_{2}^{-1} F_{1}\left(\begin{array}{c}
-k, N+k \\
2
\end{array} ; x^{2}\right) .
\end{gathered}
$$

The following formula gives the eigenvalues of the generator of the $O_{N}^{*}$-invariant semigroup on the half-liberated sphere $S_{*}^{N-1}$ associated to the pair $b=1$ and $\nu=0$. By analogy with the classical sphere, these values can be considered as the eigenvalues of the Laplace operator of the half-liberated sphere (up to a rescaling by $N-1$, see Remark (7.15). 
Corollary 7.11. For any $k \geq 0$,

$$
\begin{aligned}
\left(q_{2 k}^{*}\right)^{\prime}(1) & =\frac{2 k(N+k-1)}{N-1} \\
\left(q_{2 k+1}^{*}\right)^{\prime}(1) & =\frac{(2 k+1) N+2 k^{2}-1}{N-1} .
\end{aligned}
$$

Proof. $q_{0}^{\prime}(1)=0$ is obvious.

For $k \geq 1$, by the equation (7.1), we have

$$
\begin{aligned}
& \left(q_{2 k}^{*}\right)^{\prime}(1)=\left.\frac{2 x \frac{d}{d x^{2}}{ }_{2} F_{1}\left(\begin{array}{c}
-k, N+k-1 \\
1
\end{array} x^{2}\right)}{{ }_{2} F_{1}\left(\begin{array}{c}
-k, N+k-1 \\
1
\end{array} ; 1\right)}\right|_{x=1} \\
& =\frac{-2 k(N+k-1){ }_{2} F_{1}\left(\begin{array}{c}
-k+1, N+k \\
2
\end{array} ;\right)}{{ }_{2} F_{1}\left(\begin{array}{c}
-k, N+k-1 \\
1
\end{array}\right)} \\
& =-2 k(N+k-1) \frac{\Gamma(2) \Gamma(1-N)}{\Gamma(k+1) \Gamma(2-N-k)} \frac{\Gamma(k+1) \Gamma(2-N-k)}{\Gamma(1) \Gamma(2-N)} \\
& =\frac{2 k(N+k-1)}{N-1} \text {; } \\
& \left(q_{2 k+1}^{*}\right)^{\prime}(1)=\left.\frac{\frac{d}{d x}\left(x \cdot{ }_{2} F_{1}\left(\begin{array}{c}
-k, N+k \\
2
\end{array} x^{2}\right)\right)}{{ }_{2} F_{1}\left(\begin{array}{c}
-k, N+k \\
2
\end{array} 1\right)}\right|_{x=1} \\
& =\left.\frac{{ }_{2} F_{1}\left(\begin{array}{c}
-k, N+k \\
2
\end{array} x^{2}\right)+x \cdot\left(2 x \frac{d}{d x^{2}}{ }_{2} F_{1}\left(\begin{array}{c}
-k, N+k \\
2
\end{array} ; x^{2}\right)\right)}{{ }_{2} F_{1}\left(\begin{array}{c}
-k, N+k \\
2
\end{array} 1\right)}\right|_{x=1} \\
& =\frac{{ }_{2} F_{1}\left(\begin{array}{c}
-k, N+k \\
2
\end{array} ; 1\right)+2 \cdot \frac{-k(N+k)}{2}{ }_{2} F_{1}\left(\begin{array}{c}
-k+1, N+k+1 \\
3
\end{array} ; 1\right)}{{ }_{2} F_{1}\left(\begin{array}{c}
-k, N+k \\
2
\end{array} 1\right)} \\
& =\frac{(2 k+1) N+2 k^{2}-1}{N-1} \text {. }
\end{aligned}
$$

7.3. The free sphere $S_{+}^{N-1}$. Finally, we consider about the free case.

In fact, due to the asymptotic semicircle law of $\sqrt{N+2} u_{11}$ when $N \rightarrow \infty$ [BCZJ09], we expect that $q_{s}^{+}(x) \rightarrow U_{s}(\sqrt{N} x) / \sqrt{N^{s}}$, where $U_{s}(x)$ is the $s^{\text {th }}$ Chebyshev polynomial of the second kind. Therefore, $\lim _{N \rightarrow \infty} q_{s}^{+}(x)=x^{s}$. So for the special case where the generating functional $\psi$ is associated to the pair $b=1, \nu=0$, the eigenvalues for the subspace $D_{s}$ converge as $N \rightarrow \infty$, $\lim _{N \rightarrow \infty} \lambda_{s}=-\left(x^{s}\right)^{\prime}(1)=-s$. We now derive relations between polynomials $\left(q_{s}^{+}\right)_{s \geq 0}$ for general finite $N$.

Proposition 7.12. For any $N \in \mathbb{N}$, the orthogonal polynomials defined as above satisfy the following three-term recurrence relation:

$$
a_{s+1} q_{s+2}^{+}(x)=U_{s+1}(N) q_{s+1}^{+}(x) x-a_{s} q_{s}^{+}(x) \quad \forall s \geq 0
$$


where $q_{0}^{+}(x)=1, q_{1}^{+}(x)=x$,

$$
a_{s}=\sum_{k=0}^{s}(-1)^{s+k} U_{k}(N)= \begin{cases}U_{m}(N)\left(U_{m}(N)-U_{m-1}(N)\right) & \text { if } s=2 m, \\ U_{m}(N)\left(U_{m+1}(N)-U_{m}(N)\right) & \text { if } s=2 m+1,\end{cases}
$$

and where $U_{s}(N)$ denotes the value of the $s^{\text {th }}$ Chebyshev polynomial of the second kind at the point $N$.

Proof. For free orthogonal quantum group, the irreducible corepresentations have the following fusion rule [Ba92]:

$$
u^{(s+1)} \otimes u=u^{(s+2)} \oplus u^{(s)} .
$$

This implies that $u_{11}^{(s+1)} u_{11} \in V_{s+2} \oplus V_{s}$. Applying the two-sided conditional expectation $\mathbb{E}_{b i}^{\Phi}$ to both sides, we see that $u_{11}^{(s+1)}$. $u_{11}$ can be written as the linear combination of $u_{11}^{(s+2)}$ and $u_{11}^{(s)}$.

Let $\lambda_{s}$ be a number such that the coefficient of the highest degree of the polynomial $\lambda_{s} q_{s}^{+}(x)$ is 1 . Since $q_{s}^{+}(1)=1$, we have

$$
\lambda_{s+2} q_{s+2}^{+}(x)=\lambda_{s+1} q_{s+1}^{+}(x) x-\left(\lambda_{s+1}-\lambda_{s+2}\right) q_{s}^{+}(x) .
$$

By the orthogonality of $\left(q_{s}^{+}\left(u_{11}\right)\right)_{s \geq 0}$ and $h_{S_{+}^{N-1}}\left(\left(q_{s}^{+}\left(u_{11}\right)\right)^{2}\right)=h_{S_{+}^{N-1}}\left(\left(u_{11}^{(s)}\right)^{2}\right)=$ $1 / U_{s}(N)$, we have

$$
\begin{aligned}
0 & =h_{S_{+}^{N-1}}\left(\lambda_{s+2} q_{s+2}^{+}\left(u_{11}\right) q_{s}^{+}\left(u_{11}\right)\right) \\
& =h_{S_{+}^{N-1}}\left(\lambda_{s+1} q_{s+1}^{+}\left(u_{11}\right) q_{s}^{+}\left(u_{11}\right) u_{11}\right)-\left(\lambda_{s+1}-\lambda_{s+2}\right) h_{S_{+}^{N-1}}\left(\left(q_{s}^{+}\left(u_{11}\right)\right)^{2}\right) \\
& =\frac{\lambda_{s+1}^{2}}{\lambda_{s}} h_{S_{+}^{N-1}}\left(\left(q_{s+1}^{+}\left(u_{11}\right)\right)^{2}\right)+0-\left(\lambda_{s+1}-\lambda_{s+2}\right) h_{S_{+}^{N-1}}\left(\left(q_{s}^{+}\left(u_{11}\right)\right)^{2}\right) \\
& =\frac{\lambda_{s+1}^{2}}{\lambda_{s} U_{s+1}(N)}-\frac{\lambda_{s+1}-\lambda_{s+2}}{U_{s}(N)} .
\end{aligned}
$$

Therefore

$$
\frac{\lambda_{s+2}}{\lambda_{s+1}}=1-\frac{\lambda_{s+1}}{\lambda_{s}} \cdot \frac{U_{s}(N)}{U_{s+1}(N)} .
$$

Set $a_{s}=\frac{\lambda_{s+1}}{\lambda_{s}} \cdot U_{s}(N)$, then

$$
a_{s+1} q_{s+2}^{+}(x)=U_{s+1}(N) q_{s+1}^{+}(x) x-a_{s} q_{s}^{+}(x),
$$

and

$$
a_{s+1}=U_{s+1}(N)-a_{s} .
$$

From the latter equation we can get

$$
a_{s}=\sum_{k=0}^{s}(-1)^{s+k} U_{k}(N) \text {. }
$$


The following formula gives the eigenvalues of the generator of the $O_{N}^{+}$-invariant semigroup on the free sphere $S_{+}^{N-1}$ associated to the pair $b=1$ and $\nu=0$. By analogy with the classical sphere, these values can be considered as the eigenvalues of the Laplace operator of the free sphere (up to a rescaling by $N-1$, see Remark 7.15).

\section{Corollary 7.13.}

$$
\left(q_{s}^{+}\right)^{\prime}(1)=\sum_{r=0}^{s-1} \frac{\sum_{k=0}^{r} U_{k}(N)}{\sum_{k=0}^{r}(-1)^{r+k} U_{k}(N)} \quad \forall s \geq 1
$$

Proof. Appling Proposition 7.12 and taking derivatives on both sides, we get

$$
a_{s+1}\left(q_{s+2}^{+}\right)^{\prime}(x)=U_{s+1}(N)\left(\left(q_{s+1}^{+}\right)^{\prime}(x) x+q_{s+1}^{+}(x)\right)-a_{s}\left(q_{s}^{+}\right)^{\prime}(x) .
$$

Since $q_{s}^{+}(1)=1$, we have

$$
a_{s+1}\left(q_{s+2}^{+}\right)^{\prime}(1)=U_{s+1}(N)\left(q_{s+1}^{+}\right)^{\prime}(1)+U_{s+1}(N)-a_{s}\left(q_{s}^{+}\right)^{\prime}(1) .
$$

Rewrite this equation using $U_{s+1}(N)=a_{s+1}+a_{s}$,

$$
a_{s+1}\left(\left(q_{s+2}^{+}\right)^{\prime}(1)-\left(q_{s+1}^{+}\right)^{\prime}(1)\right)=U_{s+1}(N)+a_{s}\left(\left(q_{s+1}^{+}\right)^{\prime}(1)-\left(q_{s}^{+}\right)^{\prime}(1)\right) .
$$

Therefore,

$$
a_{s}\left(\left(q_{s+1}^{+}\right)^{\prime}(1)-\left(q_{s}^{+}\right)^{\prime}(1)\right)=\sum_{k=0}^{s} U_{k}(N)
$$

This implies

$$
\left(q_{s+1}^{+}\right)^{\prime}(1)=\sum_{r=0}^{s} \frac{\sum_{k=0}^{r} U_{k}(N)}{a_{r}}
$$

We can get an estimate of these eigenvalues that grows linearly in $s$.

Corollary 7.14. For any $N \geq 2$,

$$
s \leq\left(q_{s}^{+}\right)^{\prime}(1) \leq \frac{N+2}{N-2} s, \quad \forall s \geq 0,
$$

(where the upper becomes $+\infty$ for $N=2$ ).

Proof. Using the relation $U_{s}(N) N=U_{s+1}(N)+U_{s-1}(N)$, we have

$$
\sum_{k=0}^{m} U_{2 k}(N)=\frac{1}{2}\left(U_{2 m}(N)+U_{0}(N)\right)+\frac{N}{2}\left(\sum_{k=1}^{m} U_{2 k-1}(N)\right),
$$

and

$$
\sum_{k=0}^{m} U_{2 k+1}(N)=\frac{1}{2}\left(U_{2 m+1}(N)+U_{1}(N)\right)+U_{0}(N)+\frac{N}{2}\left(\sum_{k=1}^{m} U_{2 k}(N)\right) .
$$


Therefore

$$
\frac{\sum_{k=0}^{r} U_{k}(N)}{\sum_{k=0}^{r}(-1)^{r+k} U_{k}(N)} \leq \frac{N / 2+1}{N / 2-1}
$$

Remark 7.15. For the classical sphere, we know that the Laplace operator is the operator whose eigenvector are the Jacobi polynomials $J_{s}$ and whose eigenvalues are $\lambda_{s}=s(s+N-2)=-(N-1) q_{s}^{\prime}(1)$. So the generator for classical spheres in Theorem 7.5, is induced from the generating functional $\psi$ associated to the pair $(b, \nu)=(N-1,0)$ is the Laplace operator. In the same manner, we may define the Laplace operator $\Delta_{*}$ on the half-liberated sphere and the Laplace operator $\Delta_{+}$on free sphere.

Remark 7.16. Recall that we showed in Proposition 2.3 that central convolution semigroups of states on $C^{u}(\mathbb{G})$ also induce $\mathbb{G}$-invariant Markov semigroups on any quantum space $\mathbb{X}$ equipped with a right $\mathbb{G}$-action. The generating functionals of central convolution semigroups of states on $C^{u}\left(O_{N}^{+}\right)$were classified in CFK14, Corollary 10.3]. This gives the formula

$$
\lambda_{s}=-b \frac{U_{s}^{\prime}(N)}{U_{s}(N)}+\int_{-N}^{N} \frac{U_{s}(x)-U_{s}(N)}{U_{s}(N)(N-x)} \nu(\mathrm{d} x), \quad s=0,1, \ldots
$$

with $b$ a positive real number, $\nu$ a finite positive measure on the interval $[-N, N]$ and $\left(U_{s}\right)_{s=0}^{\infty}$ the Chebyshev polynomials of the second kind defined by $U_{0}(x)=1$, $U_{1}(x)=x, U_{s+1}(x)=x U_{s}(x)-U_{s-1}(x)$ for $s \geq 1$.

Recall again that by [BCZJ09, Theorem 5.3] the distribution of $\sqrt{N+2} u_{11}$ converges uniformally to the semicircle distribution, which is the measure of orthogonality of the Chebyshev polynomials. This suggests that the eigenvalues given by Theorem 7.5 and in Equation (7.2) for the free sphere $S_{+}^{N-1}$ should be close for large $N$.

7.4. Spectral dimensions. The Weyl formula for the eigenvalues of the LaplaceBeltrami operator $\Delta_{\mathcal{M}}$ on a compact Riemannian $C^{\infty}$-manifold $(\mathcal{M}, g)$ of dimension $N$ states that

$$
N(\lambda) \sim_{\lambda \rightarrow+\infty} \frac{\lambda^{N / 2}|\mathcal{M}|}{(4 \pi)^{N / 2} \Gamma\left(\frac{N}{2}+1\right)}
$$

cf. MP49, where $|\mathcal{M}|$ denotes the volume of $(\mathcal{M}, g), N(\lambda)$ denotes the number eigenvalues of the Laplace-Beltrami operator that are less then or equal to $\lambda$, and $f \sim_{\lambda \rightarrow+\infty} g$ stands for "asymptotically equivalent," i.e., for $\lim _{\lambda \rightarrow \infty} \frac{f(\lambda)}{g(\lambda)}=1$. This implies that the zeta-function $\zeta_{M}(z)=\sum_{\lambda \in \sigma\left(\Delta_{M}\right)} m_{\lambda} \lambda^{z}$, where $m_{\lambda}$ denotes the multiplicity of the eigenvalue $\lambda$, has a simple pole in $\frac{N}{2}$, and that this value is also the abscissa of convergence of the series. For this reason, we define the "spectral dimension" $d_{L}$ of the spheres $S_{*}^{N-1}$ (w.r.t. a generator $L$ ) as the abscissa of convergence of the series $\sum_{s=0}^{\infty} m_{s} \lambda_{s}^{-z / 2}$, where $\left(\lambda_{s}\right)_{s \geq 0}$ are the eigenvalues of $L$ which we classified in Theorem 7.5. Note that this definition is equivalent to 
Connes' definition in Co04a, Co04b, if we construct a Dirac operator $D_{L}$ from $L$ as in CFK14, since the eigenvalues of $D_{L}$ will be $\left( \pm \sqrt{\lambda_{s}}\right)_{s \geq 0}$

The spectral dimension $d_{L}$ is equal to the infimum of all $d>0$ such that the $\operatorname{sum} \sum_{s} m_{s}\left(-\lambda_{s}\right)^{-d / 2}$ is finite.

For simplicity, we will only consider the special case $b=1$ and $\nu=0$ of the eigenvalues given in Theorem 7.5 .

7.4.1. The classical sphere $S^{N-1}$. By definition of $D_{s}$,

$$
\operatorname{dim} D_{s}=\operatorname{dim} H_{s}-\operatorname{dim} H_{s-1},
$$

where

$$
H_{s}=\operatorname{span}\left\{x_{1}^{k_{1}} \cdots x_{N}^{k_{N}}: k_{1}+\cdots+k_{N} \leq s\right\}
$$

Since $x_{1}^{2}=1-\sum_{i=2}^{N} x_{i}^{2}$, we only need consider $k_{1}=0$ or $k_{1}=1$ in above formula.

Recall that $\left|\left\{\left(k_{1}, k_{2}, \cdots, k_{n}\right) \in \mathbb{N}^{n}: k_{1}+k_{2}+\cdots k_{n}=M\right\}\right|=\left(\begin{array}{c}M+n-1 \\ n-1\end{array}\right)$.

For $k_{1}=0$,

$$
\operatorname{dim} \operatorname{span}\left\{x_{2}^{k_{2}} \cdots x_{N}^{k_{N}}: k_{2}+\cdots+k_{N}=s\right\}=\left(\begin{array}{c}
s+N-2 \\
N-2
\end{array}\right)
$$

and for $k_{1}=1$,

$$
\operatorname{dim} \operatorname{span}\left\{x_{1} x_{2}^{k_{2}} \cdots x_{N}^{k_{N}}: k_{2}+\cdots+k_{N}=s-1\right\}=\left(\begin{array}{c}
s+N-3 \\
N-2
\end{array}\right)
$$

therefore,

$$
m_{s}=\operatorname{dim} D_{s}=\left(\begin{array}{c}
s+N-2 \\
N-2
\end{array}\right)+\left(\begin{array}{c}
s+N-3 \\
N-2
\end{array}\right) \asymp s^{N-2} .
$$

where the notation $a_{s} \asymp b_{s}$ for two sequences of strictly positive numbers means that they are of the same order of magnitude. More precisely, $a_{s} \asymp b_{s}$ means that there exist constants $c, C>0$ such that for all $s \in \mathbb{N}, c a_{s} \leq b_{s} \leq C a_{s}$.

For the eigenvalues we have $-\lambda_{s}=\frac{s(s+N-2)}{N-1} \asymp s^{2}$, and therefore we find $d_{L}=$ $N-1$, as expected.

7.4.2. The half-liberated sphere $S_{*}^{N-1}$. Again, $\operatorname{dim} D_{s}=\operatorname{dim} H_{s}-\operatorname{dim} H_{s-1}$. Consider first the even case, i.e. $s=2 m$.

Let $X=x_{\ell_{1}} x_{\ell_{2}} \cdots x_{\ell_{2 m-1}} x_{\ell_{2 m}} \in D_{2 m}$. Use black dots "•" for odd positions and white dots "o" for even positions, i.e., associate the diagram

$$
\bullet \circ \bullet \circ \cdots \bullet \circ \text {. }
$$

to the monomial $X$. Since we have the relation $x_{\ell_{1}} x_{\ell_{2}} x_{\ell_{3}}=x_{\ell_{3}} x_{\ell_{2}} x_{\ell_{1}}$ for the generators, we can freely permute the generators $x_{\ell_{2 k-1}}$ that are placed on black dots “•" (i.e., in odd positions) among each other. Similarly, generators $x_{\ell_{2 k}}$ sitting on white dots "०" (i.e., in even positions) can be permuted among each other.. 
Write $x_{\ell_{2 k-1}}=a_{i_{k}}$ and $x_{\ell_{2 k}}=b_{j_{k}}$, respectively, for the generators on black and white dots, then $X=a_{i_{1}} b_{j_{1}} \cdots a_{i_{m}} b_{j_{m}}$.

Since $a_{i_{k}}$ is commute among each other, we set $a_{i_{1}} a_{i_{2}} \cdots a_{i_{m}}=x_{1}^{p_{1}} x_{2}^{p_{2}} \cdots x_{N}^{p_{N}}$ with $p_{1}+p_{2}+\cdots p_{N}=m$. Similary, set $b_{i_{1}} b_{i_{2}} \cdots b_{i_{m}}=x_{1}^{q_{1}} x_{2}^{q_{2}} \cdots x_{N}^{q_{N}}$ with $q_{1}+$ $q_{2}+\cdots q_{N}=m$.

Since $x_{1}^{2}=1-\sum_{i=2}^{N} x_{i}^{2}$, we can assume $p_{1}=0$ or $q_{1}=0$. Indeed, if both monomials $a_{i_{1}} a_{i_{2}} \cdots a_{i_{m}}$ and $b_{i_{1}} b_{i_{2}} \cdots b_{i_{m}}$ contain the generator $x_{1}$, then we can we could move $x_{1}$ to the first position in both the subwords $a_{i_{1}} a_{i_{2}} \cdots a_{i_{m}}$ and $b_{i_{1}} b_{i_{2}} \cdots b_{i_{m}}$, and replace the resulting $x_{1}^{2}$ by $1-\sum_{i=2}^{N} x_{i}^{2}$. In this way get one monomial that is in $H_{s-2}$, and in the remaining terms the powers of $x_{1}$ in both subwords are reduced by 1 . Iterating this procedure we can express $X$ as a linear combination of monomials which have $p_{1}=0$ or $q_{1}=0$.

Therefore,

$$
\begin{aligned}
& \operatorname{dim} D_{s}=\operatorname{dim} \operatorname{span}\left\{x_{2}^{p_{2}} \cdots x_{N}^{p_{N}}: \sum_{k=2}^{N} p_{k}=m\right\} \cdot \operatorname{dim} \operatorname{span}\left\{x_{2}^{q_{2}} \cdots x_{N}^{q_{N}}: \sum_{k=2}^{N} q_{k}=m\right\} \\
& +\operatorname{dimspan}\left\{x_{2}^{p_{2}} \cdots x_{N}^{p_{N}}: \sum_{k=2}^{N} p_{k}=m\right\} \cdot \operatorname{dim} \operatorname{span}\left\{x_{1}^{q_{1}} x_{2}^{q_{2}} \cdots x_{N}^{q_{N}}: q_{1}>0, \sum_{k=1}^{N} q_{k}=m\right\} \\
& +\operatorname{dim} \operatorname{span}\left\{x_{1}^{p_{1}} x_{2}^{p_{2}} \cdots x_{N}^{p_{N}}: p_{1}>0, \sum_{k=1}^{N} p_{k}=m\right\} \cdot \operatorname{dim} \operatorname{span}\left\{x_{2}^{q_{2}} \cdots x_{N}^{q_{N}}: \sum_{k=2}^{N} q_{k}=m\right\} \\
& =\left(\begin{array}{c}
m+N-2 \\
N-2
\end{array}\right)^{2}+2\left(\begin{array}{c}
m+N-2 \\
N-2
\end{array}\right)\left(\begin{array}{c}
m+N-2 \\
N-1
\end{array}\right) \asymp m^{2 N-3} \asymp s^{2 N-3} .
\end{aligned}
$$

Similary, when $s=2 m+1$,

$$
\begin{aligned}
\operatorname{dim} D_{s}= & \left(\begin{array}{c}
m+N-2 \\
N-2
\end{array}\right)\left(\begin{array}{c}
m+N-1 \\
N-2
\end{array}\right)+\left(\begin{array}{c}
m+N-1 \\
N-2
\end{array}\right)\left(\begin{array}{c}
m+N-1 \\
N-1
\end{array}\right) \\
& +\left(\begin{array}{c}
m+N \\
N-1
\end{array}\right)\left(\begin{array}{c}
m+N-2 \\
N-2
\end{array}\right) \\
& \asymp s^{2 N-3} .
\end{aligned}
$$

On the other hand, by Corollary $7.11,-\lambda_{s} \asymp s^{2}$. Hence,

$$
d_{L}=2(N-1) \text {. }
$$

Banica showed in [Ba16, Theorem 1.14]. that $C\left(S_{*}^{N-1}\right)$ can be embedded into the $\mathrm{C}^{*}$-algebra $M_{2}\left(C\left(S_{\mathbb{C}}^{N-1}\right)\right)$ of continuous functions with values in $2 \times 2$-matrices on the complex sphere $S_{\mathbb{C}}^{N-1}=\left\{z=\left(z_{1}, \ldots, z_{N}\right) \in \mathbb{C}^{N}: \sum_{i=1}^{N}\left|z_{i}\right|^{2}=1\right\}$. This embedding sends the generators $x_{i}, i=1, \ldots, N$, to the functions $\pi\left(x_{i}\right): S_{\mathbb{C}}^{N-1} \ni$ $z=\left(z_{1}, \ldots, z_{N}\right) \mapsto\left(\begin{array}{cc}0 & z_{i} \\ \bar{z}_{i} & 0\end{array}\right)$. Evaluating these functions in a point $z \in S_{\mathbb{C}}^{N-1}$ defines a unique 2-dimensional representation $\pi_{z}: C\left(S_{\mathbb{C}}^{N-1}\right) \rightarrow M_{2}(\mathbb{C})$. Two 
of these 2-dimensional representations $\pi_{z}$ and $\pi_{w}, z, w \in S_{\mathbb{C}}^{N-1}$, are unitarily equivalent if and only if there exists a complex number $\lambda$ with $|\lambda|=1$ such that $z=\lambda w$. This means that the embedding passes to the projective complexe sphere $P_{\mathbb{C}}^{N-1}=S_{\mathbb{C}}^{N-1} / \sim$, where $\sim$ is the equivalence relation on $S_{\mathbb{C}}^{N-1}$ defined by

$$
z_{1} \sim z_{2} \Leftrightarrow \exists \lambda \in \mathbb{C}, z_{1}=\lambda z_{2}
$$

Since the dimension of $P_{\mathbb{C}}^{N-1}$ as a real manifold is $2(N-1)$, this provides a heuristic explanation for the value of the spectral dimension $d_{L}$ for the halfliberated sphere $S_{*}^{N-1}$.

7.4.3. The free sphere $S_{+}^{N-1}$. For the free case, $D_{s}=\operatorname{span}\left\{u_{1 i}^{(s)}: 1 \leq i \leq d_{s}\right\}$ where $u_{i j}^{(s)}$ are the cofficients of the $s^{\text {th }}$ irreducible corepresentation $u^{(s)}$, which has dimension $d_{s}=U_{s}(N)$.

Let us first consider the case $N=2$. Since $U_{s}(2)=s+1$, we get $a_{s}=[s / 2]+1$ and $\sum_{k=0}^{r}=\frac{(r+1)(r+2)}{2}$. By Corollary 7.14, we have

$$
\begin{aligned}
-\lambda_{2 k+1} & =\left(q_{2 k+1}^{+}\right) \prime(1)=2 k^{2}+4 k+1, \\
-\lambda_{2 k} & =\left(q_{2 k}^{+}\right) \prime(1)=2 k^{2}+2 k .
\end{aligned}
$$

Therefore,

$$
-\lambda_{s} \asymp s^{2}, \quad m_{s} \asymp s .
$$

This implies $d_{L}=2$ for $N=2$. For $N=2$, the defining relation of the free sphere $S_{+}^{2}$ can be written as $x_{2}^{2}=1-x_{1}^{2}$, which implies $x_{1} x_{2}^{2}=x_{2}^{2} x_{1}$, as well as the other half-commutation relations $x_{i} x_{j} x_{k}=x_{k} x_{j} x_{i}, i, j, k \in\{1,2\}$. So we have $C^{u}\left(S_{+}^{2}\right) \cong C^{u}\left(S_{*}^{2}\right)$, i.e., the free and the half-liberated two-dimensional spheres coincide.

By Corollary 7.14, $\lambda_{s} \asymp s$ for $N \geq 3$. Furthermore, in this case $m_{s}=U_{s}(N) \asymp$ $N^{s}$. Hence,

$$
d_{L}=\left\{\begin{array}{cc}
2 & \text { if } N=2 \\
+\infty & \text { if } N \geq 3
\end{array}\right.
$$

This resembles the computation in [CFK14, Remark 10.4], where we found

$$
d_{D}=\left\{\begin{array}{cc}
3 & \text { if } N=2 \\
+\infty & \text { if } N \geq 3
\end{array}\right.
$$

for the spectral dimension of a spectral triple constructed from a central generating functional on the free orthogonal quantum group $O_{N}^{+}$.

\section{ACKNOWLEDGEMENTS}

We thank Teo Banica, Adam Skalski, and Haonan Zhang for fruitful discussions and useful suggestions.

UF and XW were supported by the French "Investissements d'Avenir" program, project ISITE-BFC (contract ANR-15-IDEX-03). XW was supported by the China Scholarship Council. We also acknowledge support by the French 
MAEDI and MENESR and by the Polish MNiSW through the Polonium programme.

\section{REFERENCES}

[BaM18] Dominique Bakry, L. Mbarki, The Markov sequence problem for the Jacobi polynomials and on the simplex, Preprint hal-01811880, 2018.

[Ba92] Teo Banica. Théorie des représentations du groupe quantique compact libre $O(n)$, C. R. Acad. Sci. Paris Ser. I Math., 322(1992), 241-244

[Ba16] Teo Banica. Quantum isometries, noncommutative spheres, and related integrals. In: "Topological quantum groups" Lectures from the Graduate School held in Bȩdlewo, June 28-July 11, 2015. Uwe Franz, Adam Skalski and Piotr Sołtan (eds.), Banach Center Publications, 111. Polish Academy of Sciences, Institute of Mathematics, Warsaw, 2017.

[BCZJ09] Teo Banica, Benoit Collins, and P. Zinn-Justin. Spectral analysis of the free orthogonal matrix, Int. Math. Res. Not. 17 (2009), 3286-3309.

[BG10] Teo Banica, Debashish Goswami. Quantum isometries and noncommutative spheres. Comm. Math. Phys. 298 (2010), no. 2, 343-356.

[BSS12] Teodor Banica, Adam Skalski, Piotr Sołtan. Noncommutative homogeneous spaces: The matrix case, J. Geom. Phys. 62(6), 1451-1466, 2012.

[BS09] Teodor Banica, Roland Speicher, Liberation of orthogonal Lie groups. Adv. Math. 222, No. 4, 1461-1501 (2009).

[BMT01] Erik Bédos, Gerald J. Murphy, Lars Tuset. Co-amenability of compact quantum groups. J. Geom. Phys. 40(2): 129-153, 2001/

[BMT03] Erik Bédos, Gerald J. Murphy, Lars Tuset. Amenability and co-amenability of algebraic quantum groups II. J. Funct. Anal. 201 (2003), 303-340.

[Bo54] Salomon Bochner. Positive Zonal Functions on Spheres. Proceedings of the National Academy of Sciences of the United States of America, 1954, 40(12):1141-1147.

[Br12] Michael Brannan. Approximation properties for free orthogonal and free unitary quantum groups. J. Reine Angew. Math. (Crelles Journal) 672: 223-251, 2012.

[ChV99] Yu.A. Chapovsky, L.I. Vainerman. Compact quantum hypergroups. J. Operator Theory 41 (1999), no. 2, 261-289.

[CFK14] Fabio Cipriani, Uwe Franz, Anna Kula. Symmetries of Lévy processes on compact quantum groups, their Markov semigroups and potential theory. J. Funct. Anal. 266 (2014), no. 5, 2789-2844.

[CS03] Fabio Cipriani, Jean-Luc Sauvageot. Derivations as square roots of Dirichlet forms. J. Funct. Anal. 201, No. 1, 78-120 (2003).

[DC16] Kenny De Commer. Actions of compact quantum groups In: "Topological quantum groups" Lectures from the Graduate School held in Będlewo, June 28-July 11, 2015. Uwe Franz, Adam Skalski and Piotr Sołtan (eds.), Banach Center Publications, 111. Polish Academy of Sciences, Institute of Mathematics, Warsaw, 2017.

[Co04a] Alain Connes. Cyclic cohomology, noncommutative geometry and quantum group symmetries. Noncommutative geometry, 1-71, Lecture Notes in Math., 1831, Springer, Berlin, 2004.

[Co04b] Alain Connes. Cyclic cohomology, quantum group symmetries and the local index formula for $S U_{q}(2)$. J. Inst. Math. Jussieu 3, (2004), no. 1, 17-68.

[D16] Vladimir Dobrev, Invariant differential operators. Volume 1: Noncompact semisimple Lie algebras and groups, De Gruyter Studies in Mathematical Physics 35, De Gruyter, 2016. 
[D17] Vladimir Dobrev, Invariant differential operators. Volume 2: Quantum groups, De Gruyter Studies in Mathematical Physics 39, De Gruyter 2017.

[FLS16] Uwe Franz, Hunhee Lee, Adam Skalski. Integration over the quantum diagonal subgroup and associated Fourier-like algebras, Int. J. Math. Vol. 27, 1650073 (2016), DOI 10.1142/S0129167X16500737.

[FS09a] Uwe Franz, Adam Skalski. A new characterisation of idempotent states on finite and compact quantum groups. C. R. Math. Acad. Sci. Paris 347 (2009), no. 17-18, 991-996.

[FS09b] Uwe Franz, Adam Skalski. On idempotent states on quantum groups. J. Alg. 322 (2009), no. 5, 1774-1802.

[FS00] Uwe Franz, Michael Schürmann. Lévy processes on quantum hypergroups. In "Infinite dimensional harmonic analysis (Kyoto, 1999)", pp. 93-114, Gräbner, Altendorf, 2000.

[KK16] Paweł Kasprzak, F. Khosravi, Piotr Sołtan. Integrable actions and quantum subgroups, preprint arXiv:1603.06084, 2016.

[L04] Ming Liao. Lévy processes in Lie groups. Cambridge Tracts in Mathematics, 162. Cambridge University Press, Cambridge, 2004.

[L15] Ming Liao. Convolution of probability measures on Lie groups and homogenous spaces. Potential Anal. 43 (2015), no. 4, 707-715.

[L18] Ming Liao. Invariant Markov processes under Lie group actions. Springer, 2018.

[MM17] Hiroshi Maehara, Horst Martini. Geometric probability on the sphere. Jahresber. Dtsch. Math.-Ver. 119 (2017), no. 2, 93-132.

[MP49] S. Minakshisundaram, A. Pleijel. Some properties of the eigenfunctions of the Laplaceoperator on Riemannian manifolds. Canadian J. Math. 1, (1949), 242-256.

[MVD98] Ann Maes, Alfons Van Daele, Notes on compact quantum groups. Nieuw Arch. Wiskd., IV. Ser. 16, No. 1-2, pp. 73-112 (1998).

[P87] Piotr Podleś. Quantum spheres. Lett. Math. Phys. 14 (1987), no. 3, 193-202.

[P95] Piotr Podleś. Symmetries of quantum spaces. Subgroups and quotient spaces of quantum SU(2) and SO(3) groups. Comm. Math. Phys. 170 (1995), no. 1, 1-20.

[SS16] Pekka Salmi, Adam Skalski. Idempotent states on locally compact quantum groups II. The Quarterly Journal of Mathematics (2016):1-11.

[T08] Thomas Timmermann, An invitation to quantum groups and duality. From Hopf algebras to multiplicative unitaries and beyond. EMS Textbooks in Mathematics, European Mathematical Society, 2008.

[VDW96] Van Daele, Alfons; Wang, Shuzhou Universal quantum groups. (English) Zbl 0870.17011 Int. J. Math. 7, No. 2, 255-263 (1996).

[W98] Shuzhou Wang, Quantum symmetry groups of finite spaces. Commun. Math. Phys. 195, No. 1, 195-211 (1998).

[W80] Stanisław L. Woronowicz, Pseudospaces, pseudogroups and Pontrjagin duality. In: Mathematical problems in theoretical physics, Proc. int. Conf., Lausanne 1979, Lect. Notes Phys. 116, 407-412 (1980).

[W87] Stanisław L. Woronowicz, Compact matrix pseudogroups. Commun. Math. Phys. 111, 613-665 (1987) Articles in this Issue

[W98] Stanisław L. Woronowicz, Compact quantum groups. In: Connes, A. (ed.) et al., Quantum symmetries/ Symétries quantiques. Proceedings of the Les Houches summer school, Session LXIV, Les Houches, France, August 1 - September 8, 1995. NorthHolland, 1998.

[Zh18] Haonan Zhang. Infinitely divisible states on finite quantum groups. Preprint arXiv:1809.04417v1, September 2018. 
Instytut Matematyczny, Uniwersytet WrocŁawski, Pl.Grunwaldzki 2/4, 50384 Wroceaw, Poland

E-mail address: biswarup.das@math.uni.wroc.pl

Laboratoire de mathématiques de Besançon, Université de Bourgogne FrancheComté, 16, route de Gray, F-25030 Besançon cedex, France

E-mail address: uwe.franz@univ-fcomte.fr

$U R L:$ http://lmb.univ-fcomte.fr/uwe-franz

Laboratoire de mathématiques de Besançon, Université de Bourgogne FrancheComté, 16, route de Gray, F-25 030 Besançon cedex, France

E-mail address: xumin.wang@univ-fcomte.fr 